\title{
A PARTIAL CHECKLIST OF MOTHS (LEPIDOPTERA) OF DEHRADUN, MUSSOORIE AND DEVALSARI IN GARHWAL, UTTARAKHAND, INDIA
}

ISSN 0974-7907 (Online) ISSN 0974-7893 (Print)

\section{OPEN ACCESS}

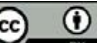

Yash Sondhi ${ }^{1} \&$ Sanjay Sondhi ${ }^{2}$

${ }^{1}$ Indian Institute of Science Education and Research, CET Campus, Thiruvananthapuram, Kerala 695016, India

${ }^{1,2}$ Titli Trust, Villa 49, Rajpur Road Enclave, Dhoran Khas, Dehradun, Uttarakhand 248001, India ${ }^{1}$ yashsondhi@gmail.com (corresponding author), 22sanjay.sondhi1@gmail.com

Abstract: Two-hundred-and-forty-eight species of moths were recorded during surveys conducted over 40 nights in Dehradun and Mussoorie in Dehradun District and Devalsari in Tehri Garhwal District in Uttarakhand.

Keywords: Biodiversity, Erebidae, Geometridae, range extension, western Himalaya.

DOI: http://dx.doi.org/10.11609/jott.2814.8.5.8756-8776 | ZooBank: urn:Isid:zoobank.org:pub:BOF270EA-2D25-4AF2-80A5-61F63356E0FE

Editor: lan J. Kitching, Natural History Museum, London, U.K.

Date of publication: 26 May 2016 (online \& print)

Manuscript details: Ms \# 3084 | Received 14 May 2015 | Final received 17 March 2016 | Finally accepted 10 April 2016

Citation: Sondhi, Y. \& S. Sondhi (2016). A partial checklist of moths (Lepidoptera) of Dehradun, Mussoorie and Devalsari in Garhwal, Uttarakhand, India. Journal of Threatened Taxa 8(5): 8756-8776; http://dx.doi.org/10.11609/jott.2814.8.5.8756-8776

Copyright: (c) Sondhi \& Sondhi 2016. Creative Commons Attribution 4.0 International License. JoTT allows unrestricted use of this article in any medium, reproduction and distribution by providing adequate credit to the authors and the source of publication.

Funding: Funding for field work was provided by Titli Trust.

Conflict of Interest: The authors declare no competing interests.

Author Details: YASH SONDHI is an integrated Masters student at Indian Institute of Science Education and Research (IISER) Trivandrum in his $4^{\text {th }}$ year. He has been interested on Lepidoptera taxonomy since his childhood and is now focusing on taxonomy and behavior of Indian moths. He is also a member of Titli Trust, Dehradun. SANJAY SONDHI is a Dehradun-based naturalist, and Founder Trustee, Titli Trust, Dehradun (www.titlitrust.com). He works on conservation research and action, and conservation education in the Himalaya, with a special focus on Lepidoptera.

Author Contribution: YS conducted the initial field work, primarily in 2011 and 2012, surveyed and identified the moths, wrote the manuscript and prepared the tables, plates and figures. SS in addition to assisting in the initial surveys also conducted surveys in 2013 and 2014, identified the moths and reviewed the manuscript.

Acknowledgements: The authors wish to acknowledge the support received from the Moths of India email group, including the members lan Kitching and Peter Smetacek for help with moth identifications. Additionally, we wish to thank the reviewers who provided some useful comments on previous versions of this manuscript; Anchal Sondhi, who assisted in the field work; Dr. Dhananjai Mohan, APCCF, Uttarakhand Forest Department and Dr. Karthik Vasudevan, LACONES, Centre for Cellular and Molecular Biology, Hyderabad, for their inputs designing the study; and Dr. Arun Pratap Singh, Forest Research Institute, Dehradun, for his suggestions and assistance in obtaining some of the required moth related literature.
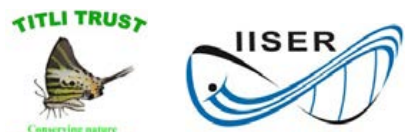


\section{INTRODUCTION}

The moth diversity of Uttarakhand is poorly studied. Although Smetacek (1994, 2008, 2009, 2011) has studied moths in the Nainital District in Kumaon for more than 30 years, studies in Garhwal are limited. Roonwal et al. (1963) listed the Lepidoptera fauna in the entomological collection of the Forest Research Institute, which has numerous moth species collected from Garhwal. Arora et al. (1977) collected moths during a Swiss Expedition and listed 45 moth species. Arora (1997) also reported on moth surveys from Nanda Devi Biosphere Reserve. More recently, Sanyal et al. (2013) and Uniyal et al. (2013) studied the entomofauna of the Gangotri landscape, listing 468 moth species. The present study, conducted in Dehradun, Mussoorie and the surrounding areas, over a four-year period through systematic and opportunistic surveys, seeks to build further on the meagre published information of the moths of the Garhwal region.

\section{MATERIALS AND METHODS}

The partial checklist of moths presented in this paper is based on both systematic and opportunistic surveys that were conducted primarily in Dehradun, Mussoorie, and Devalsari over a period of four years, 2011-2014, with forty nights of survey covering all months except January and April (Table 2). Moths were not collected but were primarily identified using digital colour photographs.

The study sites (Table 1) for the systematic surveys were Malsi Range Colony, Danda Lokhand, Dhoran Khas and Welham Girls' School, in and around Dehradun City. In addition, moths were opportunistically surveyed within Rajaji National Park at the following sites: Chilla, Rasulpur and Asarodi. In Mussoorie, moths were surveyed at the Padmini Nivas Hotel, Jabberkhet and Bata Ghat near Woodstock School. All these locations are in the Dehradun District, Uttarakhand. Devalsari Reserved Forest, which is $50 \mathrm{~km}$ from Mussoorie in Tehri Garhwal District, was also surveyed for moths. Other than Jabberkhet and Bata Ghat in Mussoorie, which were surveyed exclusively by day, all other localities were surveyed primarily at night using a 160W mercury vapour bulb and a white cloth screen, although there are several records from random sampling during the day from these locations. The study sites are shown in Fig. 1.

Available literature was used to identify moths, which included Moore (1882), Hampson (1892-1896), Bell \& Scott (1937), Barlow (1982), Holloway (1983-
Table 1. Overview of survey sites

\begin{tabular}{|c|c|c|}
\hline Study site & GPS coordinates & $\begin{array}{l}\text { Altitude } \\
(\mathrm{m})\end{array}$ \\
\hline \multicolumn{3}{|l|}{ Dehradun } \\
\hline Dhoran Khas & $30^{\circ} 21^{\prime} 39.10^{\prime \prime} \mathrm{N} \& 78^{\circ} 4^{\prime} 58.67^{\prime \prime} \mathrm{E}$ & 794 \\
\hline Danda Lokhand & $30^{\circ} 21^{\prime} 33.84^{\prime \prime} \mathrm{N} \& 78^{\circ} 5^{\prime} 32.28^{\prime \prime} \mathrm{E}$ & 802 \\
\hline Malsi & $30^{\circ} 22^{\prime} 42.26^{\prime \prime} \mathrm{N} \& 78^{\circ} 4^{\prime} 5.00^{\prime \prime} \mathrm{E}$ & 824 \\
\hline Welham Girls School & $30^{\circ} 18^{\prime} 55.40^{\prime \prime} \mathrm{N} \& 78^{\circ} 3^{\prime} 22.27^{\prime \prime} \mathrm{E}$ & 673 \\
\hline \multicolumn{3}{|l|}{ Rajaji } \\
\hline Chilla & $29^{\circ} 58^{\prime} 16.02^{\prime \prime} \mathrm{N} \& 78^{\circ} 12^{\prime} 40.11^{\prime \prime} \mathrm{E}$ & 328 \\
\hline Rasulpur & $30^{\circ} 2^{\prime} 31.17^{\prime \prime} \mathrm{N} \& 77^{\circ} 57^{\prime} 47.06^{\prime \prime} \mathrm{E}$ & 333 \\
\hline Asarodi & $30^{\circ} 14^{\prime} 19.24^{\prime \prime} \mathrm{N} \& 77^{0} 57^{\prime} 57.79^{\prime \prime} \mathrm{E}$ & 760 \\
\hline \multicolumn{3}{|l|}{ Mussoorie } \\
\hline Padmini Nivas Hotel & $30^{\circ} 27^{\prime} 32.16^{\prime \prime} \mathrm{N} \& 78^{\circ} 4^{\prime} 4.64^{\prime \prime} \mathrm{E}$ & 1989 \\
\hline Jabberkhet & $30^{\circ} 27^{\prime} 14.19^{\prime \prime} \mathrm{N} \& 78^{\circ} 6^{\prime} 40.03^{\prime \prime} \mathrm{E}$ & 2105 \\
\hline Bata Ghat & $30^{\circ} 27^{\prime} 18.86^{\prime \prime} \mathrm{N} \& 78^{\circ} 5^{\prime} 59.42^{\prime \prime} \mathrm{E}$ & 2060 \\
\hline Devalsari Reserved Forest & $30^{\circ} 32^{\prime} 43.92^{\prime \prime} \mathrm{N} \& 78^{\circ} 11^{\prime} 34.44^{\prime \prime} \mathrm{E}$ & 1725 \\
\hline
\end{tabular}

Table 2. Overview of survey effort and species identified

\begin{tabular}{|l|l|c|c|}
\hline Area & Seasons & $\begin{array}{c}\text { No. survey } \\
\text { nights }\end{array}$ & $\begin{array}{c}\text { Number of moth } \\
\text { species identified }\end{array}$ \\
\hline Dhoran Khas & $\begin{array}{l}\text { February, March, May } \\
\text { June, July, August, } \\
\text { September, December }\end{array}$ & 20 & 103 \\
\hline $\begin{array}{l}\text { Danda } \\
\text { Lokhand }\end{array}$ & June, July & 4 & 58 \\
\hline Malsi & July & 4 & 71 \\
\hline Welham Girls & July, November & 5 & 13 \\
\hline Rajaji & $\begin{array}{l}\text { March, October, } \\
\text { November }\end{array}$ & 3 & 36 \\
\hline Mussoorie & July, September & 2 & 63 \\
\hline Devalsari & October & 2 & 17 \\
\hline & Total & 40 & 248 \\
\hline
\end{tabular}

2011), Haruta (1992-2000), Pinratana \& Lampe (1990), Robinson et al. (1994), Inoue et al. (1996), Allen (1993), Kendrick (2002), Kononenko \& Pinratana (2005), Zolotuhin \& Pinratana (2005), Schintlmeister \& Pinratana (2007), and Pinratana \& Cerny (2009). The classification system used by van Nieukerken et al. (2011) has been followed. Many moth species need examination of genitalia for confirmation of their identity; hence species identities that could not be confirmed only through photographs, have been provisionally identified. 


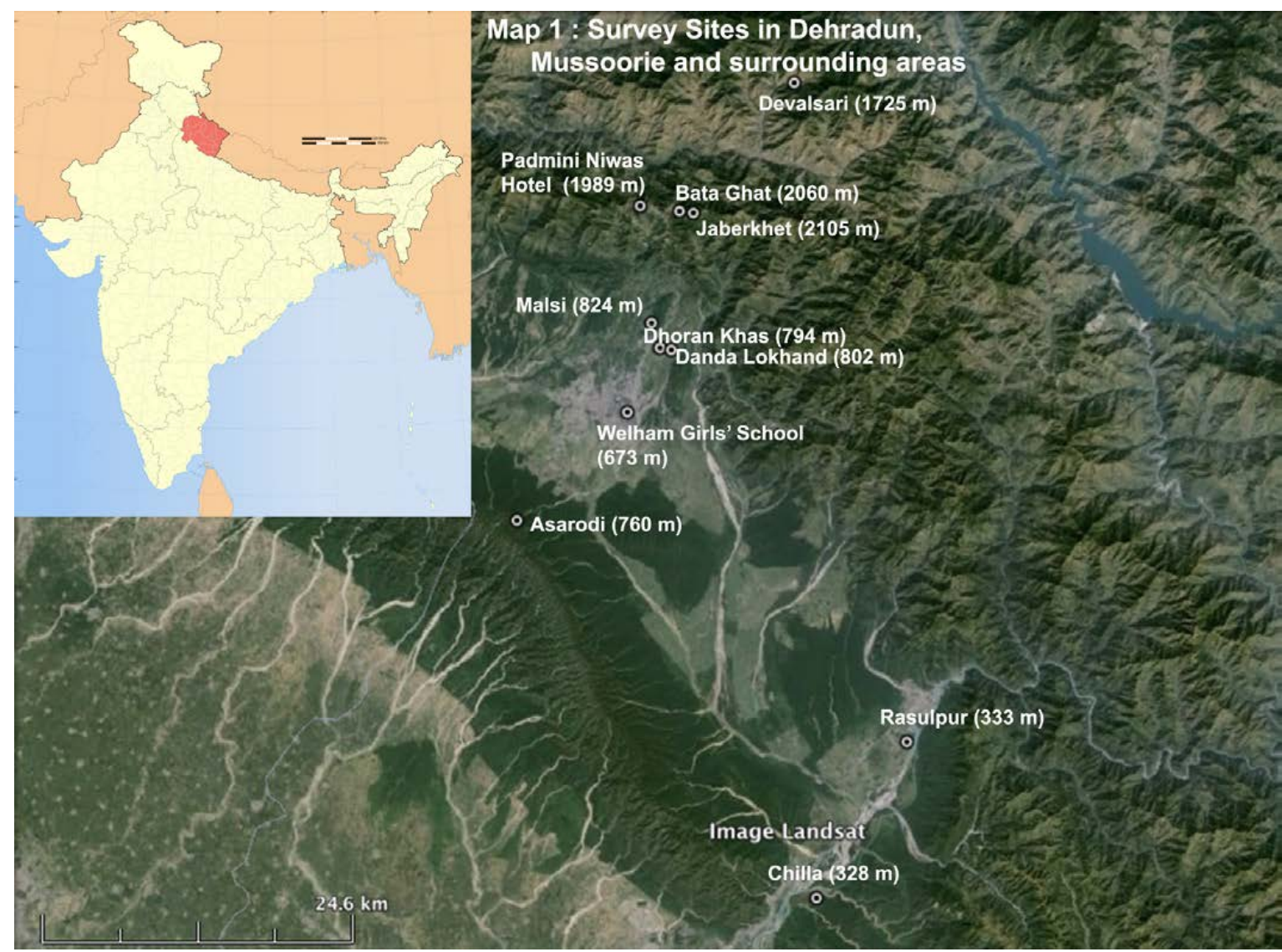

Figure 1. Study sites and surrounding areas

\section{RESULTS AND DISCUSSION}

During the study, 215 moth species were identified to the species level and another 33 to genus level (Table 3, Images 1-250). All moths in the checklist are illustrated in Images 1-250. All the photographs were taken by the two authors. Moths from the families Geometridae, Erebidae and Crambidae were the most numerous (Table 4). Some interesting records made during the survey, which are range extensions, are discussed below.

YS recorded the sphingid, Theretra griseomarginata (Hampson, 1898), from Malsi, Dehradun on 1 July 2011. The known distribution of this species is Kumaon eastwards through Nepal and northeast India (Bell 1937; Allen 1993; Smetacek 2008). There is no record of this species from Garhwal Himalaya in the Forest Research Institute collection (Roonwal et al. 1963). This represents the westernmost record for this species, extending its known range by over $200 \mathrm{~km}$, and is the first record from Garhwal Himalaya.

On 2 October 2014, SS recorded Corymica deducta caustolomaria form wirthi (Walker, 1866) at Devalsari Forest Rest House. This form was described from Kumaon
(Smetacek 2004). This species extends eastwards to eastern Himalaya (Sikkim) and hills of northeast India (Khasi Hills, Meghalaya). It has also been recorded from Kerala (Hampson 1895). This is the first record of this species and form from Garhwal Himalaya.

YS recorded a male Macotasa tortricoides (Walker, 1862) from Malsi on 22 June 2011. SS recorded the same species from Rajaji on 1 November 2011. The male is very different from other species in the genus. $M$. costalis is the only species known from this region (Pinratana \& Cerny 2009). Originally a Bornean endemic, this is the second published record for India, the only other record being from Goa (Singh et al. 2013).

YS recorded Teulisna protuberans (Moore, 1878) on 29 June 2011 from Danda Lokhand. The current Indian distribution is Sikkim, Arunachal Pradesh and West Bengal (Singh et al. 2014); it is also known from Bhutan and Thailand (Pinratana \& Cerny 2009). Previously, the only known species of this genus from northern India was $T$. nebulosa, which has very different forewing markings. This is the first record of this moth from western Himalaya and is a significant range extension.

SS recorded Anaplectoides cf. tamsi (Boursin, 1955) 
Table 3. Partial checklist of moths recorded

\begin{tabular}{|c|c|c|c|c|c|c|}
\hline & Family & Subfamily & Scientific name & Author \& Year of description & ${ }^{1}$ Location & $\begin{array}{l}{ }^{2} \text { Months } \\
\text { observed }\end{array}$ \\
\hline 1 & Eupterotidae & Eupterotinae & Eupterote bifasciata & Kishida, 1994 & $\mathrm{DL}$ & vii \\
\hline 2 & Eupterotidae & Eupterotinae & Eupterote undata form sinuata & Blanchard, 1844 & DK,DL,MA,WG & vi,vii \\
\hline 3 & Eupterotidae & Eupterotinae & Ganisa plana & Walker, 1855 & DK,MA & vi-vii \\
\hline 4 & Brahmaeidae & - & Brahmaea hearseyi & White, 1861 & $\mathrm{DL}$ & vi \\
\hline 5 & Saturniidae & - & Actias selene & Hübner, 1807 & DK & iii \\
\hline 6 & Saturniidae & - & Loepa katinka & Westwood, 1848 & $\mathrm{DL}$ & vii \\
\hline 7 & Saturniidae & - & Samia canningi & Hutton, 1859 & DK & vii \\
\hline 8 & Sphingidae & Macroglossinae & Eupanacra mydon & Walker, 1856 & DK & $x$ \\
\hline 9 & Sphingidae & Macroglossinae & Hippotion celerio & Linnaeus, 1758 & DK & v \\
\hline 10 & Sphingidae & Macroglossinae & Macroglossum belis & Linnaeus, 1758 & DK & viii \\
\hline 11 & Sphingidae & Macroglossinae & Nephele hespera & Fabricius, 1775 & DK & v,vii \\
\hline 12 & Sphingidae & Macroglossinae & Rhagastis castor & Walker, 1856 & MU & viii \\
\hline 13 & Sphingidae & Macroglossinae & Theretra alecto & Linnaeus, 1758 & DK & v \\
\hline 14 & Sphingidae & Macroglossinae & Theretra griseomarginata* & Hampson, 1898 & MA & vii \\
\hline 15 & Sphingidae & Smerinthinae & Ambulyx liturata & Butler, 1875 & $\mathrm{DL}$ & vii \\
\hline 16 & Sphingidae & Smerinthinae & Cypa pallens & Jordan, 1926 & DK & v \\
\hline 17 & Sphingidae & Sphinginae & Agrius convolvuli & Linnaeus, 1758 & DK & v \\
\hline 18 & Callidulidae & - & Callidula erycinoides & Felder, 1874 & MU & $x$ \\
\hline 19 & Cossidae & - & Zeuzera coffeae & Nietner, 1861 & RA & iii \\
\hline 20 & Drepanidae & Drepaninae & Auzata semipavonaria & Walker, 1862 & DV & $x$ \\
\hline 21 & Drepanidae & Drepaninae & Deroca cf. hyalina & Walker, 1855 & $\mathrm{DV}, \mathrm{MU}$ & viii-ix \\
\hline 22 & Drepanidae & Drepaninae & Deroca cf. hidda bifida & Watson, 1957 & $\mathrm{DV}, \mathrm{MU}$ & $x$ \\
\hline 23 & Drepanidae & Drepaninae & Macrocilix mysticata & Walker 1863 & MU & viii \\
\hline 24 & Drepanidae & Drepaninae & Nordstromia lilacina & Moore, 1888 & MU & viii \\
\hline 25 & Drepanidae & Drepaninae & Oreta extensa suffusa & Walker, 1855 & DV & $\mathrm{x}$ \\
\hline 26 & Drepanidae & Drepaninae & Oreta vatama & Moore, 1866 & DV & $x$ \\
\hline 27 & Drepanidae & Drepaninae & Teldenia vestigiata & Butler, 1880 & MU & viii \\
\hline 28 & Drepanidae & Drepaninae & Tridrepana cf. albonotata & Moore, 1879 & RA & $\mathrm{xi}$ \\
\hline 29 & Drepanidae & Thyatirinae & Euparyphasma cinereofusca & Prout, 1921 & MU & vii \\
\hline 30 & Drepanidae & Thyatirinae & Thyatira batis & Linnaeus, 1758 & MU & viii \\
\hline 31 & Geometridae & Ennominae & Anonychia diversilinea & Warren, 1897 & DV & $\mathrm{x}$ \\
\hline 32 & Geometridae & Ennominae & Biston sp. & & DK & vii \\
\hline 33 & Geometridae & Ennominae & Biston suppressaria & Guenée, 1858 & DK,RA & iii,vi \\
\hline 34 & Geometridae & Ennominae & Boarmia sp. & & MA & vii \\
\hline 35 & Geometridae & Ennominae & Cassyma deletaria & Moore, 1888 & $\mathrm{MU}$ & viii \\
\hline 36 & Geometridae & Ennominae & Chiasmia cf. nora & Walker, 1861 & DL,MA & vi-vii \\
\hline 37 & Geometridae & Ennominae & Chiasmia cf. xanthonora & Walker, 1861 & $\mathrm{DL}$ & vi-vii \\
\hline 38 & Geometridae & Ennominae & Chiasmia eleonora & Hübner, 1818 & $\mathrm{DK}, \mathrm{DL}, \mathrm{MA}$ & vii \\
\hline 39 & Geometridae & Ennominae & $\begin{array}{l}\text { Corymica deducta caustolomaria } \\
\text { form wirthi* }\end{array}$ & Walker, 1860 & DV & $x$ \\
\hline 40 & Geometridae & Ennominae & Dalima patularia & Walker, 1860 & $\mathrm{DV}, \mathrm{MU}$ & viii-ix \\
\hline 41 & Geometridae & Ennominae & Ectropidia shoreae & Prout, 1934 & $\mathrm{DK}, \mathrm{DL}$ & vi-vii \\
\hline 42 & Geometridae & Ennominae & Fascellina chromataria & Walker, 1860 & MA,MU & vii \\
\hline 43 & Geometridae & Ennominae & Fascellina plagiata & Walker, 1860 & DV & $\mathrm{x}$ \\
\hline 44 & Geometridae & Ennominae & Gasterocome pannosaria & Moore, 1867 & MU & vii \\
\hline
\end{tabular}




\begin{tabular}{|c|c|c|c|c|c|c|}
\hline & Family & Subfamily & Scientific name & Author \& Year of description & ${ }^{1}$ Location & $\begin{array}{l}{ }^{2} \text { Months } \\
\text { observed }\end{array}$ \\
\hline 45 & Geometridae & Ennominae & Heterolocha sp. & & $\mathrm{MU}$ & ix \\
\hline 46 & Geometridae & Ennominae & Heterostegane cf. urbica urbica & Swinhoe, 1895 & $\mathrm{DK}, \mathrm{DL}, \mathrm{MA}$ & vi-viii \\
\hline 47 & Geometridae & Ennominae & Heterostegane subtessellata & Walker, 1863 & DK & vii \\
\hline 48 & Geometridae & Ennominae & Hyperythra lutea & Stoll, 1781 & $\mathrm{DL}, \mathrm{MU}$ & vii \\
\hline 49 & Geometridae & Ennominae & Hypomecis transcissa & Walker, 1866 & DK & $v$ \\
\hline 50 & Geometridae & Ennominae & Hyposidra talaca & Walker, 1860 & DK,DL,MA,WG & vi-vii \\
\hline 51 & Geometridae & Ennominae & Krananda semihyalina & Walker, 1868 & DV & $x$ \\
\hline 52 & Geometridae & Ennominae & Leptomiza calcearia & Walker, 1860 & $\mathrm{MU}$ & ix \\
\hline 53 & Geometridae & Ennominae & Medasina cf. albidaria & Walker, 1866 & $\mathrm{MU}$ & viii \\
\hline 54 & Geometridae & Ennominae & Menophra subplagiata & Walker, 1860 & $\mathrm{MU}$ & viii \\
\hline 55 & Geometridae & Ennominae & Myrioblephara albibasis & Hampson, 1895 & $\mathrm{MU}$ & viii \\
\hline 56 & Geometridae & Ennominae & Opisthograptis cf. mimulina & Butler, 1886 & DV & $x$ \\
\hline 57 & Geometridae & Ennominae & Opisthograptis moelleri & Warren, 1893 & DV & $x$ \\
\hline 58 & Geometridae & Ennominae & Ourapteryx clara & Matsumura, 1910 & $\mathrm{MU}$ & vii \\
\hline 59 & Geometridae & Ennominae & Ozola sinuicosta & Prout, 1910 & $\mathrm{DL}$ & vii \\
\hline 60 & Geometridae & Ennominae & Pachyodes cf. erionoma & Swinhoe, 1893 & $\mathrm{MU}$ & viii \\
\hline 61 & Geometridae & Ennominae & Parapholodes fuliginea & Hampson, 1891 & DK & $x$ \\
\hline 62 & Geometridae & Ennominae & Percnia belluaria & Guenée, 1858 & DV & $x$ \\
\hline 63 & Geometridae & Ennominae & Percnia maculata & Moore, 1867 & DV & $x$ \\
\hline 64 & Geometridae & Ennominae & Petelia cf. immaculata & Hampson, 1893 & DK & vii \\
\hline 65 & Geometridae & Ennominae & Pingasa cf. pseudoterpnaria & Guenée, 1858 & DK & vi \\
\hline 66 & Geometridae & Ennominae & Psyra angulifera & Walker, 1867 & DV & $x$ \\
\hline 67 & Geometridae & Ennominae & Thinopteryx crocoptera & Kollar, 1844 & $\mathrm{MU}$ & vii \\
\hline 68 & Geometridae & Ennominae & Thinopteryx nebulosa & Butler, 1883 & $\mathrm{MU}$ & viii \\
\hline 69 & Geometridae & Ennominae & Xandrames dholaria & Moore, 1868 & DV & $x$ \\
\hline 70 & Geometridae & Ennominae & Zamarada sp. & & $\mathrm{DL}, \mathrm{DK}, \mathrm{MU}, \mathrm{RA}$ & vi-viii,xi \\
\hline 71 & Geometridae & Geometrinae & Comibaena cassidara & Guenée, 1857 & DK & $v$ \\
\hline 72 & Geometridae & Geometrinae & Hemithea cf. constipunctata & Moore, 1867 & $\mathrm{DK}, \mathrm{DV}, \mathrm{MA}$ & vi-vii, $x$ \\
\hline 73 & Geometridae & Geometrinae & Hemithea ochrolauta & Warren, 1894 & $\mathrm{MU}$ & viii \\
\hline 74 & Geometridae & Geometrinae & Mixochlora vittata & Moore, 1867 & $\mathrm{MU}$ & $x$ \\
\hline 75 & Geometridae & Geometrinae & Ornithospila avicularia & Guenée, 1857 & $\mathrm{RA}, \mathrm{DK}, \mathrm{MA}$ & $v i, x i$ \\
\hline 76 & Geometridae & Geometrinae & Prasinocyma near delicata & Warren, 1912 & MA & vi \\
\hline 77 & Geometridae & Geometrinae & Protuliocnemis biplagiata & Moore, 1887 & DK & vii \\
\hline 78 & Geometridae & Geometrinae & $\begin{array}{l}\text { Tanaorhinus reciprocatus } \\
\text { confuciarius }\end{array}$ & Walker, 1861 & $\mathrm{MU}$ & vii \\
\hline 79 & Geometridae & Geometrinae & Thalassodes cf. veraria & Guenée, 1857 & $\mathrm{DK}, \mathrm{DK}, \mathrm{MU}$ & vi-vii \\
\hline 80 & Geometridae & Larentiinae & Ecliptopera triangulifera & Moore, 1888 & $\mathrm{MU}$ & viii \\
\hline 81 & Geometridae & Larentiinae & Electrophaes cf. fulgidaria & Leech, 1897 & RA & $\mathrm{xi}$ \\
\hline 82 & Geometridae & Oenchrominae & Eumelia rosalia & Stoll, 1781 & RA & $x$ \\
\hline 83 & Geometridae & Sterrhinae & Problepsis vulgaris & Butler, 1889 & $\mathrm{DK}, \mathrm{DL}$ & v,vii \\
\hline 84 & Geometridae & Sterrhinae & Rhodometra sacraria & Linnaeus, 1767 & $\mathrm{DK}, \mathrm{DL}, \mathrm{MA}$ & vi-vii \\
\hline 85 & Geometridae & Sterrhinae & Scopula sp. & & $\mathrm{MA}, \mathrm{MU}$ & vi-vii \\
\hline 86 & Geometridae & Sterrhinae & Somatina cf. plynusaria & Walker, 1862 & DK & vii \\
\hline 87 & Geometridae & Sterrhinae & Timandra correspondens & Hampson, 1895 & DV & $x$ \\
\hline 88 & Geometridae & Sterrhinae & Traminda mundissima & Walker, 1861 & $\mathrm{DL}, \mathrm{MU}, \mathrm{WG}$ & vii \\
\hline 89 & Uraniidae & Epipleminae & Epiplema bicaudata & Moore, 1867 & $\mathrm{MU}$ & vii \\
\hline
\end{tabular}




\begin{tabular}{|c|c|c|c|c|c|c|}
\hline & Family & Subfamily & Scientific name & Author \& Year of description & ${ }^{1}$ Location & $\begin{array}{l}{ }^{2} \text { Months } \\
\text { observed }\end{array}$ \\
\hline 90 & Lasiocampidae & - & Gastropacha sp. & & DK & ii \\
\hline 91 & Lasiocampidae & - & Syrastrena minor & Moore, 1879 & MA & vi \\
\hline 92 & Lasiocampidae & - & Trabala vishnou & Lefèbvre, 1827 & DK, DV & $\mathrm{iii}, \mathrm{x}$ \\
\hline 93 & Lasiocampidae & - & Kunugia cf. lattipennis & Walker, 1855 & MA & vi \\
\hline 94 & Erebidae & Aganainae & Asota caricae & Fabricius, 1775 & $\begin{array}{l}\text { DK, DL, MA, RA, } \\
\text { WG }\end{array}$ & iii,vi-vii \\
\hline 95 & Erebidae & Aganainae & Asota ficus & Fabricius, 1775 & $\mathrm{DK}, \mathrm{MU}$ & iii,vii \\
\hline 96 & Erebidae & Agarastinae & Episteme adulatrix & Kollar, 1844 & DK & ix \\
\hline 97 & Erebidae & Arctiinae & Aemene sp. & & $\mathrm{DK}, \mathrm{DL}$ & vi \\
\hline 98 & Erebidae & Arctiinae & Alphaea imbuta & Walker, 1855 & MU & vii \\
\hline 99 & Erebidae & Arctiinae & Amata cysseus & Stoll, 1782 & DK & iii \\
\hline 100 & Erebidae & Arctiinae & Areas galactina & Hoeven, 1840 & DK,DL,MA & vi-vii \\
\hline 101 & Erebidae & Arctiinae & Argina argus & Kollar, 1844 & DK & ii-iii \\
\hline 102 & Erebidae & Arctiinae & Asura dasara & Moore, 1859 & $\mathrm{DK}, \mathrm{DL}, \mathrm{DV}, \mathrm{MU}$ & vi-vii,x \\
\hline 103 & Erebidae & Arctiinae & Barsine dharma & Moore, 1879 & $\mathrm{DL}, \mathrm{MU}, \mathrm{DK}$ & vii \\
\hline 104 & Erebidae & Arctiinae & Asura nubifascia & Walker, 1865 & MU,DV & vii, ix,x \\
\hline 105 & Erebidae & Arctiinae & Barsine cf. maculifasciata & Hampson, 1894 & MA & vii \\
\hline 106 & Erebidae & Arctiinae & Barsine linga & Moore, 1859 & $\mathrm{DK}, \mathrm{MU}$ & vi-viii \\
\hline 107 & Erebidae & Arctiinae & Barsine orientalis bigamica & Cerny, 2009 & $\mathrm{DK}, \mathrm{DL}, \mathrm{DV}, \mathrm{MA}$ & vi-vii,x \\
\hline 108 & Erebidae & Arctiinae & Chrysorabdia biridana & Walker, 1864 & DV & $x$ \\
\hline 109 & Erebidae & Arctiinae & Chrysorabdia bivitta & Walker, 1856 & $\mathrm{DV}, \mathrm{MU}$ & viii, $x$ \\
\hline 110 & Erebidae & Arctiinae & Creatonotos gangis & Linnaeus, 1763 & $\mathrm{DK}, \mathrm{DL}, \mathrm{MA}$ & iii, vii \\
\hline 111 & Erebidae & Arctiinae & Creatonotos transiens & Walker, 1855 & $\mathrm{DK}, \mathrm{DL}, \mathrm{MA}, \mathrm{WG}$ & vi-vii \\
\hline 112 & Erebidae & Arctiinae & Cyana arama & Moore, 1859 & $\mathrm{DV}, \mathrm{MU}$ & $i x-x$ \\
\hline 113 & Erebidae & Arctiinae & Cyana coccinea & Moore, 1878 & DK,MA,RA & vi-vii,x-xi \\
\hline 114 & Erebidae & Arctiinae & Cyana cf. distincta babui & Kishida, 1993 & $\mathrm{MU}$ & viii \\
\hline 115 & Erebidae & Arctiinae & Cyana cf. guttifera & Walker, 1856 & $\mathrm{DK}, \mathrm{MA}$ & vii \\
\hline 116 & Erebidae & Arctiinae & Cyana detrita & Walker, 1854 & MU & $\mathrm{xi}$ \\
\hline 117 & Erebidae & Arctiinae & Cyana gelida & Walker, 1854 & DL,MA & vi \\
\hline 118 & Erebidae & Arctiinae & Cyana puella & Drury, 1773 & $\mathrm{DL}, \mathrm{MU}, \mathrm{DK}$ & vi-viii \\
\hline 119 & Erebidae & Arctiinae & Eilema cf. costalis & Moore, 1878 & $\mathrm{DK}, \mathrm{MA}$ & vii \\
\hline 120 & Erebidae & Arctiinae & Eilema cf. calamaria. & Moore, 1878 & $\mathrm{DL}, \mathrm{DK}$ & vii \\
\hline 121 & Erebidae & Arctiinae & Eilema sp.1 & & DL,DK & vi-vii \\
\hline 122 & Erebidae & Arctiinae & Eilema sp. 2 & & MU & vii \\
\hline 123 & Erebidae & Arctiinae & Eressa confinis & Walker, 1854 & DK & vi \\
\hline 124 & Erebidae & Arctiinae & Lemyra sp. & & $\mathrm{DK}, \mathrm{MA}$ & vi \\
\hline 125 & Erebidae & Arctiinae & Lyclene cf. obsoleta & Moore, 1878 & MA & vi \\
\hline 126 & Erebidae & Arctiinae & Lyclene euprepioides & Walker, 1862 & DK & v \\
\hline 127 & Erebidae & Arctiinae & Lyclene sp. & & $\mathrm{DK}, \mathrm{MA}$ & vi-vii \\
\hline 128 & Erebidae & Arctiinae & Machrobrochis gigas & Walker, 1854 & DK & vi \\
\hline 129 & Erebidae & Arctiinae & Machrobrochis prasena & Moore, 1859 & DL,MA & vi-vii \\
\hline 130 & Erebidae & Arctiinae & Macotasa tortricoides* & Walker, 1862 & $\mathrm{MA}, \mathrm{RA}$ & vi,xi \\
\hline 131 & Erebidae & Arctiinae & Olepa ricini & Fabricius, 1775 & WG,DK & vii \\
\hline 132 & Erebidae & Arctiinae & Pangora distorta & Moore, 1879 & DK & vii \\
\hline 133 & Erebidae & Arctiinae & Rajendra perrotteti & Guérin-Ménéville, 1844 & DK & vi \\
\hline
\end{tabular}




\begin{tabular}{|c|c|c|c|c|c|c|}
\hline & Family & Subfamily & Scientific name & Author \& Year of description & ${ }^{1}$ Location & $\begin{array}{l}{ }^{2} \text { Months } \\
\text { observed }\end{array}$ \\
\hline 134 & Erebidae & Arctiinae & Sidyma albifinis & Walker, 1856 & $\mathrm{MU}$ & vii \\
\hline 135 & Erebidae & Arctiinae & Spilosoma multiguttata & Walker, 1855 & $\mathrm{MU}$ & vii \\
\hline 136 & Erebidae & Arctiinae & Spilosoma sp.1 & & $\mathrm{DL}, \mathrm{MA}, \mathrm{MU}$ & vi \\
\hline 137 & Erebidae & Arctiinae & Spilosoma sp.2 & & MA & vii \\
\hline 138 & Erebidae & Arctiinae & Syntomoides imaon & Cramer, 1780 & $\begin{array}{l}\text { DK,DV,MA, } \\
\text { RA,WG }\end{array}$ & $\mathrm{iii}, \mathrm{vi}, \mathrm{viii}, \mathrm{x}, \mathrm{x}$ \\
\hline 139 & Erebidae & Arctiinae & Teulisna cf. protuberans* & Moore, 1878 & $\mathrm{DL}$ & vi \\
\hline 140 & Erebidae & Arctiinae & Trichaeta teneiformis & Walker, 1856 & $\mathrm{MU}$ & viii \\
\hline 141 & Erebidae & Arctiinae & Utethesia lotrix & Cramer, 1777 & DK & xii \\
\hline 142 & Erebidae & Arctiinae & Vamuna remelana & Moore, 1865 & $\mathrm{DV}, \mathrm{MU}$ & $i x, x$ \\
\hline 143 & Erebidae & Arctiinae & Vamuna virilis & Rothschild, 1913 & DV & $x$ \\
\hline 144 & Erebidae & Aventiinae & Zurobata sp. 1 & & MA & vi \\
\hline 145 & Erebidae & Aventiinae & Zurobata sp. 2 & & $\mathrm{MA}$ & vi \\
\hline 146 & Erebidae & Aventiinae & Zurobata sp. 3 & & DK & xi \\
\hline 147 & Erebidae & Calpinae & Dierna strigata & Moore, 1867 & DK & vii \\
\hline 148 & Erebidae & Calpinae & Diomea lignocolora & Walker, 1857 & $\mathrm{DL}$ & vii \\
\hline 149 & Erebidae & Calpinae & Oraesia cf. emarginata & Fabricius, 1794 & RA & xi \\
\hline 150 & Erebidae & Calpinae & Calyptra cf. parva & Bänziger, 1979 & MA & vii \\
\hline 151 & Erebidae & Erebinae & Achaea janata & Linnaeus, 1758 & DK & viii-ix \\
\hline 152 & Erebidae & Erebinae & Artena dotata & Fabricius, 1794 & $\mathrm{MU}$ & viii \\
\hline 153 & Erebidae & Erebinae & Dysgonia stuposa & Fabricius, 1794 & $\mathrm{DL}, \mathrm{MA}$ & vi \\
\hline 154 & Erebidae & Erebinae & Erebus caprimulgus & Fabricius, 1775 & DK & vii \\
\hline 155 & Erebidae & Erebinae & Erebus hieroglyphica & Drury, 1773 & DK & xi \\
\hline 156 & Erebidae & Erebinae & Fodina cf. stola & Guenée, 1852 & $\mathrm{DL}$ & vi \\
\hline 157 & Erebidae & Erebinae & Fodina pallula & Guenée, 1852 & $\mathrm{DL}$ & vi \\
\hline 158 & Erebidae & Erebinae & Lygniodes hypoleuca & Guenée, 1852 & DK & v \\
\hline 159 & Erebidae & Erebinae & Mocis undata & Fabricius, 1775 & DK & iii \\
\hline 160 & Erebidae & Erebinae & Ophiusa coronata & Fabricius, 1775 & DK & vii-viii \\
\hline 161 & Erebidae & Erebinae & Ophiusa tirhaca & Cramer, 1777 & $\mathrm{MU}$ & viii \\
\hline 162 & Erebidae & Erebinae & Spirama cf. retorta & Clerck, 1759 & DK & $v$ \\
\hline 163 & Erebidae & Erebinae & Thyas juno & Dalman, 1823 & $\mathrm{MU}$ & viii \\
\hline 164 & Erebidae & Erebinae & Trigonodes hyppasia & Cramer, 1779 & DK & xii \\
\hline 165 & Erebidae & Hypeninae & Hypena cf. iconicalis & Walker, 1859 & $\mathrm{DL}$ & vii \\
\hline 166 & Erebidae & Hypeninae & Hypena quadralis & Walker, 1859 & $\mathrm{DL}, \mathrm{DK}$ & viii \\
\hline 167 & Erebidae & Hypeninae & Hypena sp. & & MA & vii \\
\hline 168 & Erebidae & Lymantriinae & Arctornis sp. 1 & & $\mathrm{MA}, \mathrm{RA}$ & vii,xi \\
\hline 169 & Erebidae & Lymantriinae & Arctornis sp. 2 & & DV,MA & vii, $x$ \\
\hline 170 & Erebidae & Lymantriinae & Arctornis-I-nigrum & Müller, 1794 & MA & vii \\
\hline 171 & Erebidae & Lymantriinae & Artaxa diagramma & Boisduval, 1844 & $\mathrm{DK}, \mathrm{DL}, \mathrm{MA}$ & vi \\
\hline 172 & Erebidae & Lymantriinae & Artaxa guttata & Walker, 1855 & $\mathrm{DL}, \mathrm{MA}, \mathrm{WG}$ & vii \\
\hline 173 & Erebidae & Lymantriinae & Dasychira sp. & & DL,MA & vii \\
\hline 174 & Erebidae & Lymantriinae & Euproctis cf. lunata & Walker, 1855 & DK & vi \\
\hline 175 & Erebidae & Lymantriinae & Euproctis cf. vitellina & Kollar, 1848 & $\mathrm{MA}$ & vii \\
\hline 176 & Erebidae & Lymantriinae & Euproctis plagiata & Walker, 1855 & MA & vii \\
\hline 177 & Erebidae & Lymantriinae & Euproctis sp. 1 & & DL,WG & vi-vii \\
\hline
\end{tabular}




\begin{tabular}{|c|c|c|c|c|c|c|}
\hline & Family & Subfamily & Scientific name & Author \& Year of description & ${ }^{1}$ Location & $\begin{array}{l}{ }^{2} \text { Months } \\
\text { observed }\end{array}$ \\
\hline 178 & Erebidae & Lymantriinae & Euproctis sp. 2 & & DL,WG,MA & vi-vii \\
\hline 179 & Erebidae & Lymantriinae & Himala argentea & Walker, 1855 & DV & $x$ \\
\hline 180 & Erebidae & Lymantriinae & Lymantria cf. concolor & Walker, 1855 & $\mathrm{DK}, \mathrm{DV}, \mathrm{RA}$ & $v i, x-x i$ \\
\hline 181 & Erebidae & Lymantriinae & Lymantria mathura & Moore, 1856 & DK,DV & $\mathrm{v}, \mathrm{x}$ \\
\hline 182 & Erebidae & Lymantriinae & Lymantria obsoleta & Walker, 1855 & MA & vii \\
\hline 183 & Erebidae & Lymantriinae & Lymantria sp. & & MA & vii \\
\hline 184 & Erebidae & Lymantriinae & Lymantria semicincta & Walker, 1855 & MA & vii \\
\hline 185 & Erebidae & Lymantriinae & Lymantriades cf. varians & Walker, 1855 & WG & vii \\
\hline 186 & Erebidae & Lymantriinae & Orgyia cf. postica & Walker, 1855 & DL,WG & vi-vii \\
\hline 187 & Erebidae & Lymantriinae & Somena cf. scintillans & Walker, 1856 & $\mathrm{DL}$ & vii \\
\hline 188 & Erebidae & Lymantriinae & Varmina indica & Walker, 1853 & MU & vii \\
\hline 189 & Erebidae & Scoliopteryginae & Anomis flava & Fabricius, 1775 & MA & vii \\
\hline 190 & Euteliidae & Euteliinae & Eutelia adulatricoides & Mell, 1943 & MU & ix \\
\hline 191 & Noctuidae & Aediinae & Aedia sp. & & $\mathrm{DK}, \mathrm{DL}$ & vii \\
\hline 192 & Noctuidae & Amphipyrinae & Callopistria cf. indica & Butler, 1891 & MA & vii \\
\hline 193 & Noctuidae & Amphipyrinae & Callyna jugaria & Walker, 1858 & $\mathrm{DL}$ & vii \\
\hline 194 & Noctuidae & Amphipyrinae & Callyna monoleuca & Walker, 1858 & MA & vii \\
\hline 195 & Noctuidae & Amphipyrinae & Chasmina judicata & Walker, 1858 & DK & vii \\
\hline 196 & Noctuidae & Amphipyrinae & Elaphria cf. conjugata & Moore, 1881 & MA & vii \\
\hline 197 & Noctuidae & Condicinae & Bagada pyrochroma & Walker, 1858 & DL,DK & vii \\
\hline 198 & Noctuidae & Noctuinae & Anaplectoides cf. tamsi* & Dierl, 1983 & DV & $\mathrm{x}$ \\
\hline 199 & Noctuidae & Plusiinae & Macdunnoughia tetragona & Walker, 1857 & DL & vii \\
\hline 200 & Noctuidae & Plusiinae & Erythroplusia sp. & & $\mathrm{DL}$ & vii \\
\hline 201 & Noctuidae & Plusiinae & Thysanoplusia intermixta & Warren, 1913 & DK & $\mathrm{iii}$ \\
\hline 202 & Noctuidae & Plusiinae & Thysanoplusia orichalcea & Fabricius, 1775 & $\mathrm{DV}, \mathrm{MU}$ & $v, x$ \\
\hline 203 & Pyralidae & Pyralinae & Endotricha olivacealis & Bremer, 1864 & MA & vii \\
\hline 204 & Pyralidae & Pyralinae & Tamaraca torridalis & Lederer, 1863 & DK & vi \\
\hline 205 & Pyralidae & Pyralinae & Xitha tactilis & Swinhoe, 1890 & $\mathrm{DK}, \mathrm{MA}$ & vi \\
\hline 206 & Crambidae & Acentropinae & Eoophyla cf. peribocalis & Walker, 1859 & $\begin{array}{l}\text { DK,DL,MA, } \\
\text { RA,WG }\end{array}$ & vii \\
\hline 207 & Crambidae & Acentropinae & Eoophyla sp. & & $\mathrm{DL}$ & vi \\
\hline 208 & Crambidae & Acentropinae & Paracymoriza vagalis & Speidel, 1984 & $\mathrm{DK}, \mathrm{DL}, \mathrm{MA}$ & vi-vii \\
\hline 209 & Crambidae & Spilomelinae & Agathodes ostentalis & Geyer, 1837 & $\mathrm{MU}$ & viii \\
\hline 210 & Crambidae & Spilomelinae & Bradina diagonalis & Guenée, 1854 & MA & vi,vii \\
\hline 211 & Crambidae & Spilomelinae & Chabula acamasalis & Walker 1859 & DK & vii \\
\hline 212 & Crambidae & Spilomelinae & Cirrhochrista brizoalis & Walker 1859 & DK & vi \\
\hline 213 & Crambidae & Spilomelinae & Cnaphalocrocis medinalis & Guenée, 1854 & MA & vii \\
\hline 214 & Crambidae & Spilomelinae & Conogethes punctiferalis & Guenée, 1854 & $\mathrm{DK}, \mathrm{DL}, \mathrm{MA}$ & vii \\
\hline 215 & Crambidae & Spilomelinae & Glyphodes bicolor & Swainson, 1821 & $\mathrm{DK}, \mathrm{DL}$ & vi-vii \\
\hline 216 & Crambidae & Spilomelinae & Hymenia cf. perspectalis & Hubner, 1790 & DK & vii \\
\hline 217 & Crambidae & Spilomelinae & Lamprosema commixta & Butler, 1879 & MU & vii \\
\hline 218 & Crambidae & Spilomelinae & Maruca cf. vitrata/testulalis & Geyer, 1832 & $\mathrm{DL}$ & vii \\
\hline 219 & Crambidae & Spilomelinae & Metoeca foedalis & Guenée, 1854 & DK & $x$ \\
\hline 220 & Crambidae & Spilomelinae & Nacloeia sp. & & MA & vii \\
\hline 221 & Crambidae & Spilomelinae & Nausinoe cf. geometralis & Guenée, 1854 & MA & vii \\
\hline
\end{tabular}




\begin{tabular}{|c|c|c|c|c|c|c|}
\hline & Family & Subfamily & Scientific name & Author \& Year of description & ${ }^{1}$ Location & $\begin{array}{l}{ }^{2} \text { Months } \\
\text { observed }\end{array}$ \\
\hline 222 & Crambidae & Spilomelinae & Nausinoe perspectata & Fabricius, 1775 & $\mathrm{DL}$ & vii \\
\hline 223 & Crambidae & Spilomelinae & Omiodes sp. & & DK,MA & vi \\
\hline 224 & Crambidae & Spilomelinae & Palpita cf. asiaticalis & Inoue, 1994 & DK & xii \\
\hline 225 & Crambidae & Spilomelinae & Parotis sp. & & DK & $x$ \\
\hline 226 & Crambidae & Spilomelinae & Piletocera cf. chyrsorycta & Meyrick, 1884 & $\mathrm{DK}, \mathrm{DL}$ & vi-vii \\
\hline 227 & Crambidae & Spilomelinae & Pilocrocis cf. barcalis & Walker, 1859 & DK & $x$ \\
\hline 228 & Crambidae & Spilomelinae & Pleuroptya cf. balteata & Fabricius , 1798 & MA & vi-vii \\
\hline 229 & Crambidae & Spilomelinae & Pleuroptya cf. deficiens & Moore, 1887 & $\mathrm{MU}$ & vii \\
\hline 230 & Crambidae & Spilomelinae & Pycnarmon cf. cribrata & Fabricius, 1794 & DK & $x$ \\
\hline 231 & Crambidae & Spilomelinae & Spoladea recurvalis & Fabricius, 1775 & DK, DV, MA, RA & $x-x i$ \\
\hline 232 & Crambidae & Spilomelinae & Syllepte concatenalis & Walker, 1856 & MA & vi \\
\hline 233 & Crambidae & Spilomelinae & Syngamia cf. falsidicalis & Walker, 1859 & MU & vii \\
\hline 234 & Crambidae & Spilomelinae & Tyspanodes linealis & Moore, 1867 & $\mathrm{DL}$ & vi \\
\hline 235 & Thyrididae & Siculodinae & Rhodonuera cf. erubrescens & Warren, 1908 & DK & $v$ \\
\hline 236 & Tineidae & - & Gerontha sp. & & MA & vii \\
\hline 237 & Oecophoridae & Oecophorinae & Periacma sp. & & MA & vi \\
\hline 238 & Tortricidae & Tortricinae & Cerace cf. cyanopyga & Diakonoff, 1950 & $\mathrm{MU}$ & ix \\
\hline 239 & Limacodidae & Limacodinae & Altha subnotata & Walker, 1865 & $\mathrm{DL}, \mathrm{MA}$ & vi-vii \\
\hline 240 & Limacodidae & Limacodinae & Cania cf. bilinea & Walker, 1855 & MA & vi \\
\hline 241 & Limacodidae & Limacodinae & Cheromettia cf. apicata & Moore, 1879 & DK & vi \\
\hline 242 & Limacodidae & Limacodinae & Miresa sp. & & DK & vi \\
\hline 243 & Limacodidae & Limacodinae & Parasa hilaris & Westwood, 1848 & MA & vii \\
\hline 244 & Limacodidae & Limacodinae & Phocoderma velutina & Kollar, 1844 & $\mathrm{DK}, \mathrm{DL}, \mathrm{MA}$ & vi-vii \\
\hline 245 & Limacodidae & Limacodinae & Thosea sp. & & MA & vii \\
\hline 246 & Zygaenidae & Chalcosiinae & Brachartona quadrimaculata & Moore, 1879 & $\mathrm{MU}$ & ix \\
\hline 247 & Zygaenidae & Chalcosiinae & Gynautocera papilionaria & Guérin-Ménéville, 1831 & RA & $x$ \\
\hline 248 & Zygaenidae & Chalcosiinae & Soritia pulchella & Kollar, 1844 & $\mathrm{MU}$ & $x$ \\
\hline
\end{tabular}

Notes: (1) Survey sites: Devalsari (DV), Danda Lokhand (DL), Dhoran Khas (DK), Malsi (MA), Mussoorie (MU), Rajaji National Park (RA), Welham Girls' School (WG). (2) Months in which the individuals were sighted. This does not exclude the possibility that the moths are not active outside these times, but is intended only to be an indicator of seasonal occurrence and flight periods. (3) Genus level identifications are represented as sp. Provisional identifications as cf. or near, the former for close matches and the latter for poor matches. "Indicates a range extension.

Table 4. Summary of families seen

\begin{tabular}{|l|c|}
\hline Family & Number of species \\
\hline Erebidae & 96 \\
\hline Geometridae & 58 \\
\hline Crambidae & 29 \\
\hline Noctuidae & 12 \\
\hline Drepanidae & 11 \\
\hline Sphingidae & 10 \\
\hline Limacodidae & 7 \\
\hline Lasiocampidae & 4 \\
\hline Assorted & 21 \\
\hline Total & 248 \\
\hline
\end{tabular}

on 3 Oct 2014 from Devalsari. There are three species in the genus which are difficult to separate without examining the genitalia, Anaplectoides inexpectata (Dierl, 1983), A. tamsi (Boursin, 1955) and A. virens (Butler, 1878). The first two species have been recorded and described from Nepal, and $A$. virens has been described from Japan but has also been recorded in Darjeeling, the Amur area and China (Dierl 1983). Although the individual recorded during the survey could be any of these three species, none of them have been previously recorded from Uttarakhand.

While this checklist is by no means comprehensive, it aims to provide an insight into the moth diversity of Dehradun and the surrounding areas, and act as a 
baseline for more detailed and comprehensive studies of the moths of this region.

\section{REFERENCES}

Allen, M.G. (1993). Marvellous moths of Nepal, Know Nepal Series Number 6. Craftsmen Press, Bangkok, 72pp.

Anonymous (2015). An identification guide of Japanese Moths. http:// www.jpmoth.org (accessed 12 March 2015)

Arora, G.S., D.K. Mandal \& M. Chaudhury (1977). On scientific result of Swiss Zoological Expedition to India, Part II. Moths of Garhwal District (north-western Himalaya). Newsletter Zoological Survey India 3: 388-395.

Arora, G.S. (1997). Insecta: Lepidoptera: Fauna of Nanda Devi Biosphere Reserve (Uttaranchal). Conservation Area Series, Zoological Survey of India 9: 67-88.

Barlow, H.S. (1982). An Introduction to the Moths of South East Asia. Malayan Nature Society, Kuala Lumpur, 305pp+50pls.

Bell, T.R.D. \& F.B. Scott (1937). The Fauna of British India, including Ceylon and Burma. Moths-Volume 5, Sphingidae. 537pp+15pls.

Dierl, W. (1983) New species of Noctuidae (Noctuinae) from Nepal and Tibet. Spixiana 6: 141-147.

Hampson, G. (1892). The Fauna of British India, including Ceylon and Burma. Moths-Volume 1, Saturniidae to Hypsidae. Taylor and Francis, London, 527pp+333figs.

Hampson, G. (1894). The Fauna of British India, including Ceylon and Burma. Moths-Volume 2, Arctiidae, Agrostidae, Noctuidae. Taylor and Francis, London, 609pp+325figs.

Hampson, G. (1895). The Fauna of British India, including Ceylon and Burma. Moths-Volume 3, Noctuidae (cont.) to Geometridae. Taylor and Francis, London, 546pp+226figs.

Hampson, G. (1896). The Fauna of British India, including Ceylon and Burma. Moths-Volume 4. Pyralidae. Taylor and Francis, London, $594 p p+287$ figs.

Haruta, T. (eds.) (1992). Moths of Nepal, Part 1, Tinea. 13 (Supplement 2). Japan Heterocerists' Society, Tokyo, $122 p p+109$ figs +32 pls.

Haruta, T. (eds.) (1993). Moths of Nepal, Part 2, Tinea. 13 (Supplement 3). Japan Heterocerists' Society, Tokyo, 160pp+221figs+32pls

Haruta, T. (eds.) (1994). Moths of Nepal, Part 3, Tinea. 14 (Supplement 1). Japan Heterocerists' Society, Tokyo, 171pp+206figs+32pls.

Haruta, T. (eds.) (1995). Moths of Nepal, Part 4, Tinea. 14 (Supplement 2). Japan Heterocerists' Society, Tokyo, 206pp+303figs $+32 \mathrm{pls}$.

Haruta, T. (eds.), (1998). Moths of Nepal, Part 5, Tinea. 15 (Supplement 1). Japan Heterocerists' Society, Tokyo, 330pp+403figs+ 32 pls.

Haruta, T. (eds.), (2000). Moths of Nepal, Part 6, Tinea. 16 (Supplement 1). Japan Heterocerists' Society, Tokyo, $163 p p+273$ figs $+14 p l s$.

Holloway, J.D. (1983). The moths of Borneo (part 4); Family Notodontidae. Malayan Nature Journal 37: 1-107.

Holloway, J.D. (1985). The moths of Borneo (part 14); Family Noctuidae: Subfamilies Euteliinae, Stictopterinae, Plusiinae, Pantheinae. Malayan Nature Journal 38: 157-317.

Holloway, J.D. (1986). The moths of Borneo (part 1); Key to families; Cossidae, Metarbelidae, Ratardidae, Dudgeoneidae, Epipyropidae and Limacodidae. Malayan Nature Journal 40: 1-165.

Holloway, J.D. (1987). The Moths of Borneo (part 3); Families Lasiocampidae, Eupterotidae, Bombycidae, Brahmaeidae, Saturniidae, Sphingidae. Southdene Sdn. Bhd., Kuala Lumpur, Malaysia, 199pp+163figs+20pls.

Holloway, J.D. (1988). The Moths of Borneo (part 6); Family Arctiidae: Subfamilies Arctiinae, Syntominae, Aganainae (to Noctuidae) Southdene Sdn. Bhd., Kuala Lumpur, Malaysia. 101pp+168fig+6pls.

Holloway, J.D. (1989). The moths of Borneo (part 12); Family Noctuidae: Subfamilies Noctuinae, Heliothinae, Hadeninae, Acronictinae, Amphipyrinae, Agaristinae. Malayan Nature Journal 43: 57-226.

Holloway, J.D. (1993). The moths of Borneo (part 11); Family
Geometridae: Subfamilies Ennominae. Malayan Nature Journal 47: 1-309pp.

Holloway, J.D. (1996). The moths of Borneo (part 9); Family Geometridae: Subfamilies Oenochrominae, Desmobathrinae, Geometrinae. Malayan Nature Journal 49: 147-326.

Holloway, J.D. (1997). The moths of Borneo (part 10); Family Geometridae: Subfamilies Sterrhinae, Larentiinae, Addenda to other subfamilies. Malayan Nature Journal 51: 1-242.

Holloway, J.D. (1999). The moths of Borneo (part 5); Family Lymantriidae. Malayan Nature Journal 53: 1-188.

Holloway, J.D. (2011). The moths of Borneo (part 2); Families Phaudidae, Himantopteridae and Zygaenidae; revised and annotated checklist. Malayan Nature Journal 63: 1-548.

Inoue, H., R.D. Kennett \& I.J. Kitching ([1996] 1997). Moths of Thailand. Volume 2, Sphingidae. Chok Chai Press. Bangkok, 149pp+ 44pls.

Kendrick, R.C. (2002). Moths (Insecta: Lepidoptera) of Hong Kong. PhD Thesis. University of Hong Kong, 660pp+47pls.

Kononenko, V.S. \& A. Pinratana (2005). Moths of Thailand, Volume 3, Noctuidae. Brothers of St. Gabriel in Thailand, 265pp.

Moore, F. (1882). List of the Lepidoptera collected by Rev. J.H. Hocking chiefly in the Kangra District, N.W. Himalayas with description of new genera and species. Proceedings of the Zoological Society of London 1882: 234-263.

Pinratana, A. \& K. Cerny (2009). Moths of Thailand, Volume 6: Arctiidae. Brothers of St. Gabriel, Thailand, 286pp+52pls.

Pinratana, A. \& R.E.J. Lampe (1990). Moths of Thailand, Volume 1, Saturniidae. Bosco Offset, Bangkok, 96pp+56pls.

Robinson, G.S., K.R. Tuck, M. Shaffer \& K. Cook (1994). The Smaller Moths of South-East Asia. Malaysian Nature Society, Kuala Lumpur, 308pp+51figs+32pls.

Roonwal, M. L., R.N. Mathur, G.D. Bhasin, P.N. Chatterjee, P.K. SenSarma, B. Singh, A. Chandra, R.S. Thapa \& K. Kumar (1963). A systematic catalogue of the main identified entomological collection at the Forest Research Institute, Dehra Dun. Indian Forest Leaflet, 121 (4), in Entomology (Lepidoptera) 31: 1295-1395.

Sanyal, A.B., V.P. Uniyal, K. Chandra \& M. Bhardwaj. (2013). Diversity and indicator species of moth (Lepidoptera: Heterocera) assemblages in different vegetation zones in Gangotri Landscape, Western Himalaya, India. ENVIS Bulletin: Wildlife and Protected Areas 14(1): 114-128.

Schintlmeister, A. \& A. Pinratana (2007). Moths of Thailand, Volume 5: Notodontidae. Brothers of St. Gabriel in Thailand, $322 \mathrm{pp}+7$ figs $+45 \mathrm{pls}$

Singh, J., N. Singh, R. Joshi (2013). First report of Macotasa tortricoides (Walker) (Arctiidae: Lithosiinae) from India along with a key to species of the genus. Journal of Entomological Research 37(4): 369-373.

Singh, J., N. Singh \& R. Joshi (2014). A checklist of subfamily Arctiinae (Erebidae : Noctuoidea : Lepidoptera) from India. Records of the Zoological Survey India (Occasional Paper) 367: 1-76.

Smetacek, P. (1994). Hawkmoths (Lepidoptera, Sphingidae) of Kumaon, N. India : A probable case of faunal Drift. Records of the Zoological Survey of India (Occasional Paper) 156: 1-55.

Smetacek, P. (2004). The genus Corymica Walker (Lepidoptera: Geometridae) in the Kumaon Himalaya with the description of a new form of $C$. deducata caustolomaria Moore. Journal of Bombay Natural History Society 101: 173-176.

Smetacek, P.(2008). Moths recorded from different elevations in Nainital District, Kumaon Himalaya, India. Bionotes 10(1): 5-15.

Smetacek, P. (2009). Additions and corrections to the list of moths recorded from different elevations in Nainital District, Kumaon Himalaya, India. Bionotes 11(4): 117-118.

Smetacek, P. (2011). Further Additions to the Moths (Lepidoptera: Heterocera) of Nainital District, Kumaon Himalaya, India. Bionotes 13(4): 140-141.

Uniyal, V.P., M. Bhardwaj \& A.K. Sanyal (2013). An Assessment of Entomofauna for Management and Conservation of Biodiversity in the Gangotri Landscape. Annual Progress Report, Wildlife Institute 

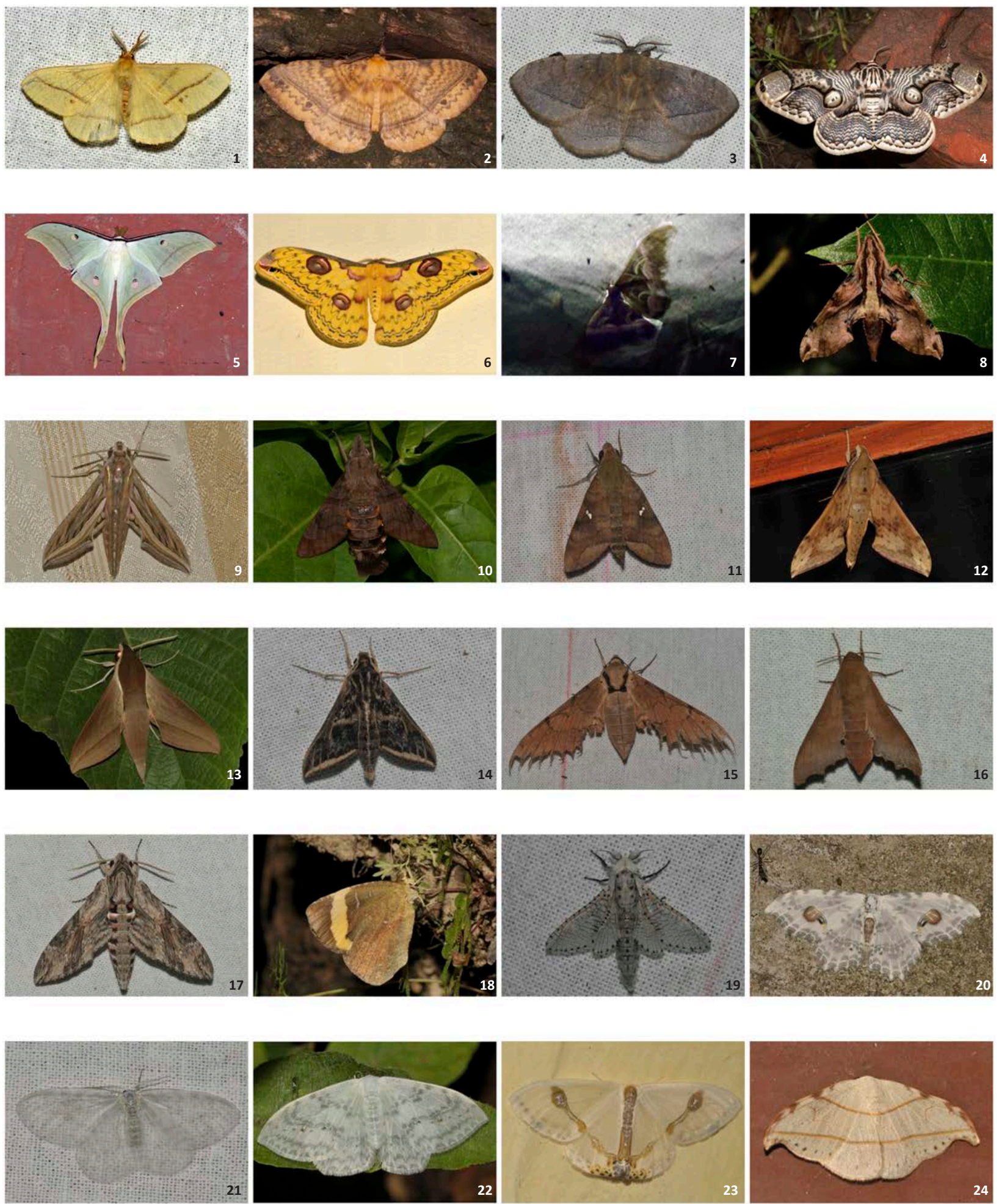

Images 1-24. 1 - Eupterote bifasciata; 2 - Eupterote undata form sinuata; 3 - Ganisa plana; 4 - Brahmaea hearseyi; 5 - Actias selene; 6 - Loepa katinka; 7 -Samia canningi; 8 - Eupanacra mydon; 9 - Hippotion celerio; 10 - Macroglossum belis; 11 - Nephele hespera;

12 - Rhagastis castor; 13 - Theretra alecto; 14 - Theretra griseomarginata"; 15 - Ambulyx liturata; 16 - Cypa pallens; 17 - Agrius convolvuli; 18 - Callidula erycinoides; 19 - Zeuzera coffeae; 20 - Auzata semipavonaria; 21 - Deroca cf. hyalina; 22 - Deroca cf. hidda bifida;

23 - Macrocilix mysticata; 24 - Nordstromia lilacina. ㄷ 5,6,7 \& 10 - Sanjay Sondhi; rest of the images (c) Yash Sondhi 

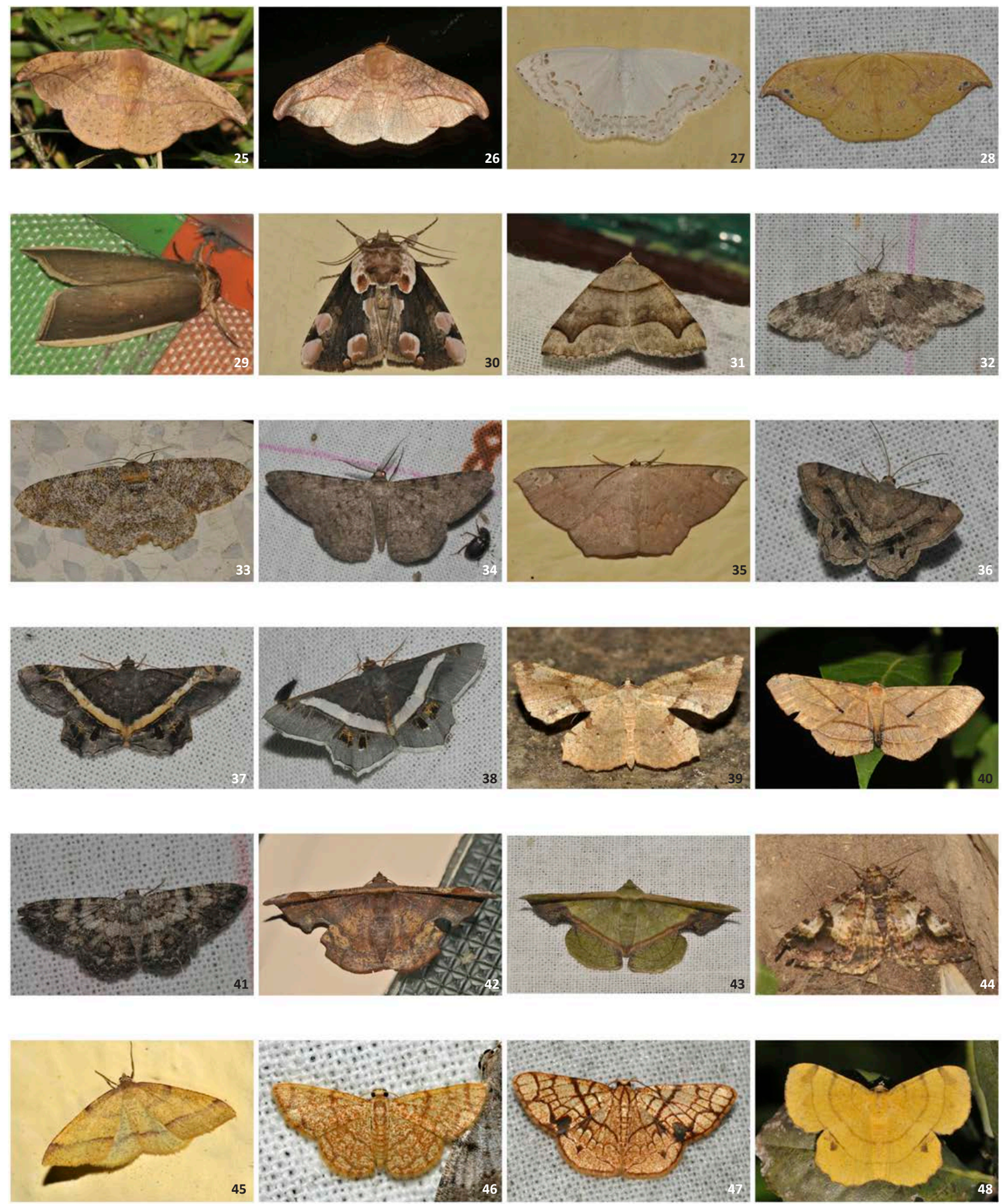

Images 25-48. 25 - Oreta extensa suffusa; 26 - Oreta vatama; 27 - Teldenia vestigiata; 28 - Tridrepana cf. albonotata; 29 - Euparyphasma cinereofusca; 30 - Thyatira batis; 31 - Anonychia diversilinea; 32 - Biston sp.; 33 - Biston suppressaria; 34 - Boarmia sp.; 35 - Cassyma deletaria; 36 - Chiasmia cf. nora; 37 - Chiasmia cf. xanthonora; 38 - Chiasmia eleonora; 39 - Corymica deducta caustolomaria form wirthi; 40 - Dalima patularia; 41 - Ectropidia shoreae; 42 - Fascellina chromataria; 43 - Fascellina plagiata; 44 - Gasterocome pannosaria; 45 - Heterolocha sp.; 46 - Heterostegane cf. urbica urbica; 47 - Heterostegane subtessellata; 48 - Hyperythra lutea. (c) 44 - Sanjay Sondhi; rest of the images (c) Yash Sondhi 

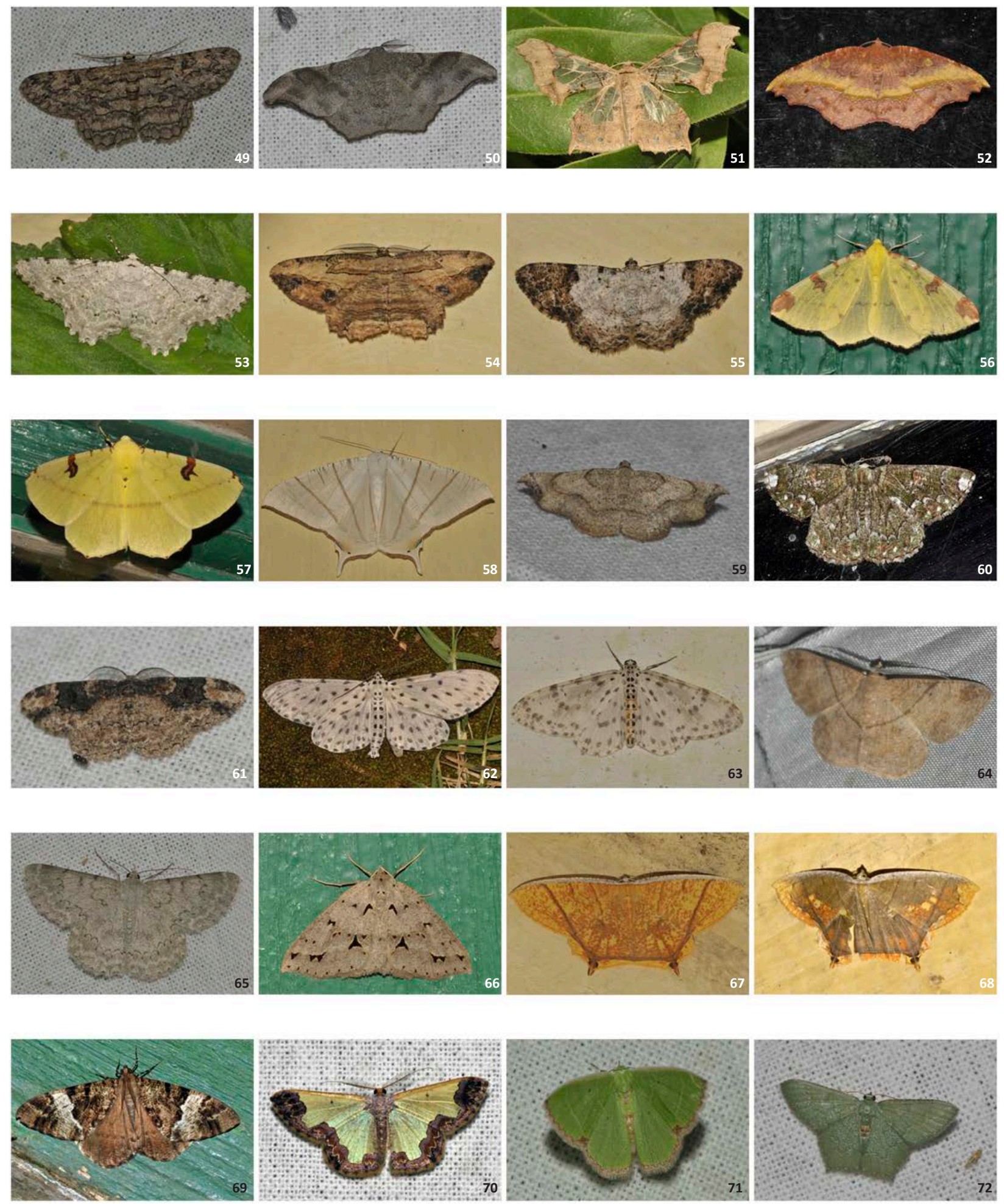

Images 49-72. 49 - Hypomecis transcissa; 50 - Hyposidra talaca; 51 - Krananda semihyalina; 52 - Leptomiza calcearia; 53 - Medasina cf. albidaria; 54 - Menophra subplagiata; 55 - Myrioblephara albibasis; 56 - Opisthograptis cf. mimulina; 57 - Opisthograptis moelleri; 58 - Ourapteryx clara; 59 - Ozola sinuicosta; 60 - Pachyodes cf. erionoma; 61 - Parapholodes fuliginea; 62 - Percnia belluaria; 63 - Percnia maculata; 64 - Petelia cf. immaculata; 65 - Pingasa cf. pseudoterpnaria; 66 - Psyra angulifera; 67 - Thinopteryx crocoptera; 68 - Thinopteryx nebulosa; 69 - Xandrames dholaria; 70 - Zamarada sp.; 71 - Comibaena cassidara; 72 - Hemithea cf. constipunctata. (c) 52 - Sanjay Sondhi; rest of the images (c) Yash Sondhi 

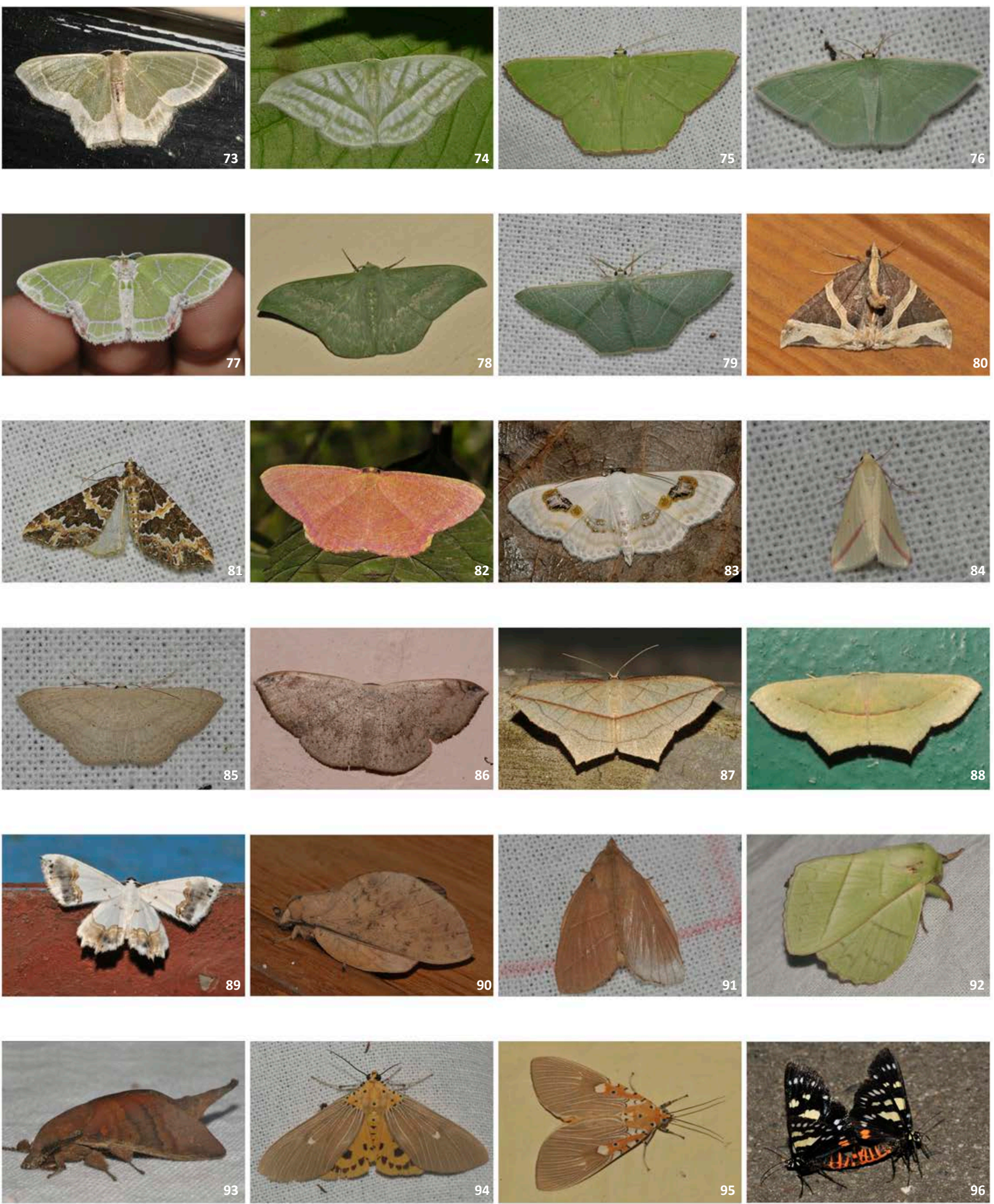

Images 73-96. 73 - Hemithea ochrolauta; 74 - Mixochlora vittata; 75 - Ornithospila avicularia; 76 - Prasinocyma near delicate; 77 - Protuliocnemis biplagiata; 78 - Tanaorhinus reciprocatus confuciarius; 79 - Thalassodes cf. veraria; 80 - Ecliptopera triangulifera; 81 - Electrophaes cf. fulgidaria; 82 - Eumelia rosalia; 83 - Problepsis vulgaris; 84 - Rhodometra sacraria; 85 - Scopula sp.; 86 - Somatina cf. plynusaria; 87 - Timandra correspondens; 88 - Traminda mundissima; 89 - Epiplema bicaudata; 90 - Gastropacha sp.; 91 - Syrastrena minor; 92 - Trabala vishnou; 93 - Kunugia cf. lattipennis; 94 - Asota caricae; 95 - Asota ficus; 96 - Episteme adulatrix.

(c) 82, 96 - Sanjay Sondhi; rest of the images (C) Yash Sondhi 

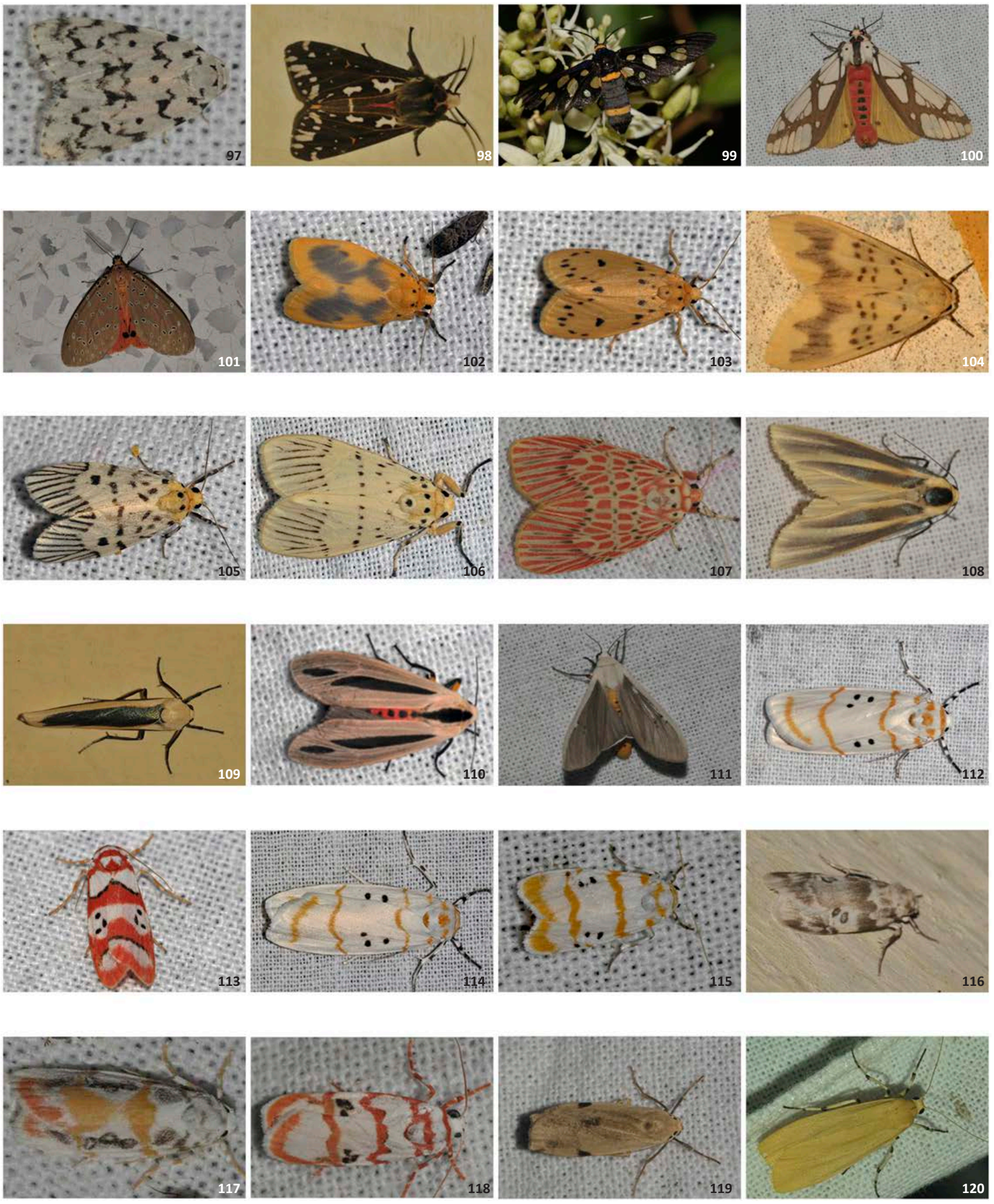

Images 97-120. 97 - Aemene sp.; 98 - Alphaea imbuta; 99 - Amata cysseus; 100 - Areas galactina; 101 - Argina argus; 102 - Asura dasara; 103 - Barsine dharma; 104 - Asura nubifascia; 105 - Barsine cf. maculifasciata; 106 - Barsine linga; 107 - Barsine orientalis bigamica; 108 - Chrysorabdia biridana; 109 - Chrysorabdia bivitta; 110 - Creatonotos gangis; 111 - Creatonotos transiens; 112 - Cyana arama; 113 - Cyana coccinea; 114 - Cyana cf. distincta babui; 115 - Cyana cf. guttifera; 116 - Cyana detrita; 117 - Cyana gelida; 118 - Cyana puella; 119 - Eilema cf. costalis; 120 - Eilema cf. calamaria. ㅇ Yash Sondhi 

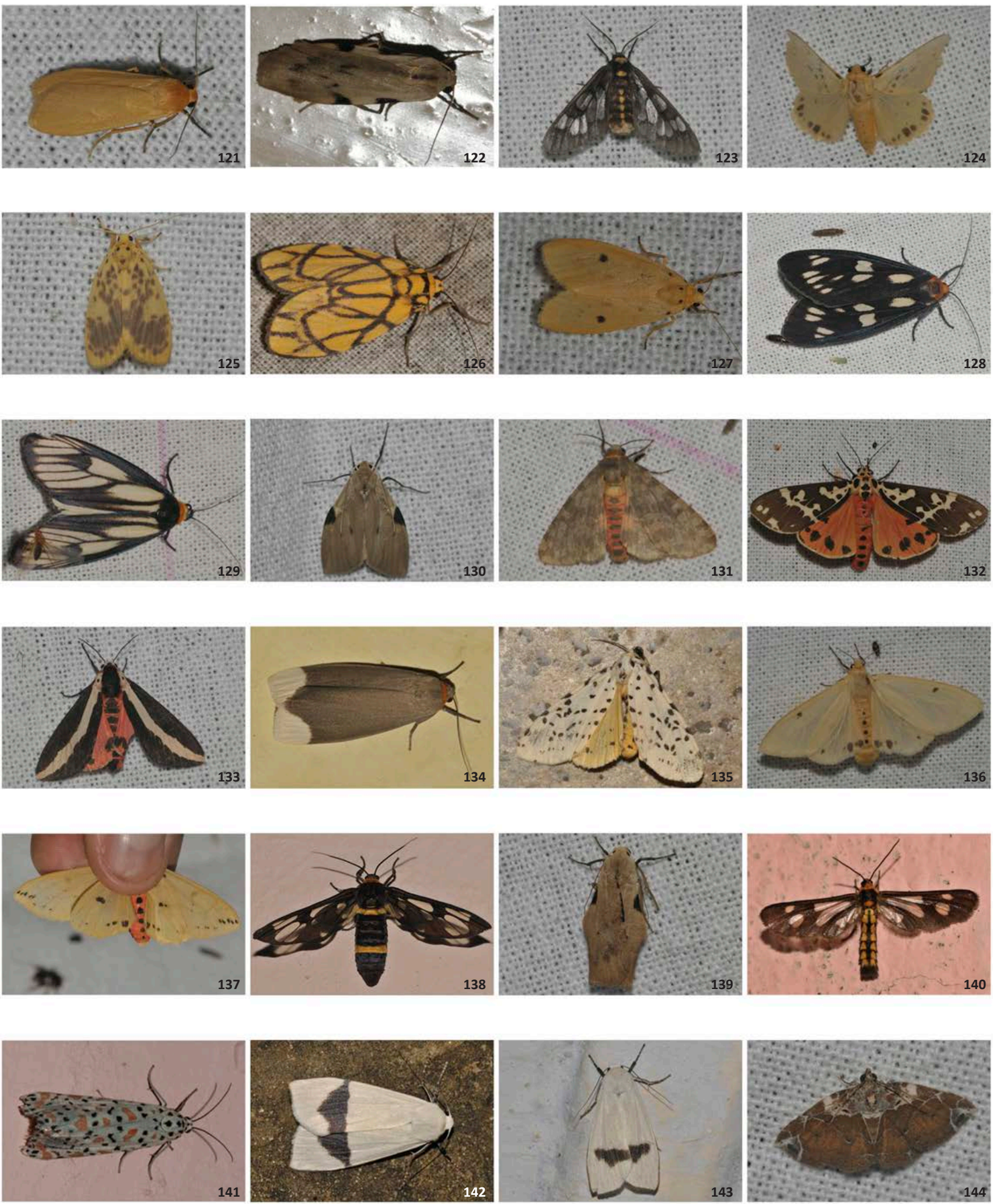

Images 121-144. 121 - Eilema sp.1; 122 - Eilema sp.2; 123 - Eressa confinis; 124 - Lemyra sp.; 125 - Lyclene cf. obsoleta; 126 - Lyclene euprepioides; 127 - Lyclene sp.; 128 - Machrobrochis gigas; 129 - Machrobrochis prasena; 130 - Macotasa tortricoides*; 131 - Olepa ricini; 132 - Pangora distorta; 133 - Rajendra perrotteti; 134 - Sidyma albifinis; 135 - Spilosoma multiguttata; 136 - Spilosoma sp.1; 137 - Spilosoma sp. 2; 138 - Syntomoides imaon; 139 - Teulisna cf. protuberans*; 140 - Trichaeta teneiformis; 141 - Utethesia lotrix; 142 - Vamuna remelana; 143 - Vamuna virilis; 144 - Zurobata sp.1. @ 140 - Sanjay Sondhi; rest of the images @ Yash Sondhi 

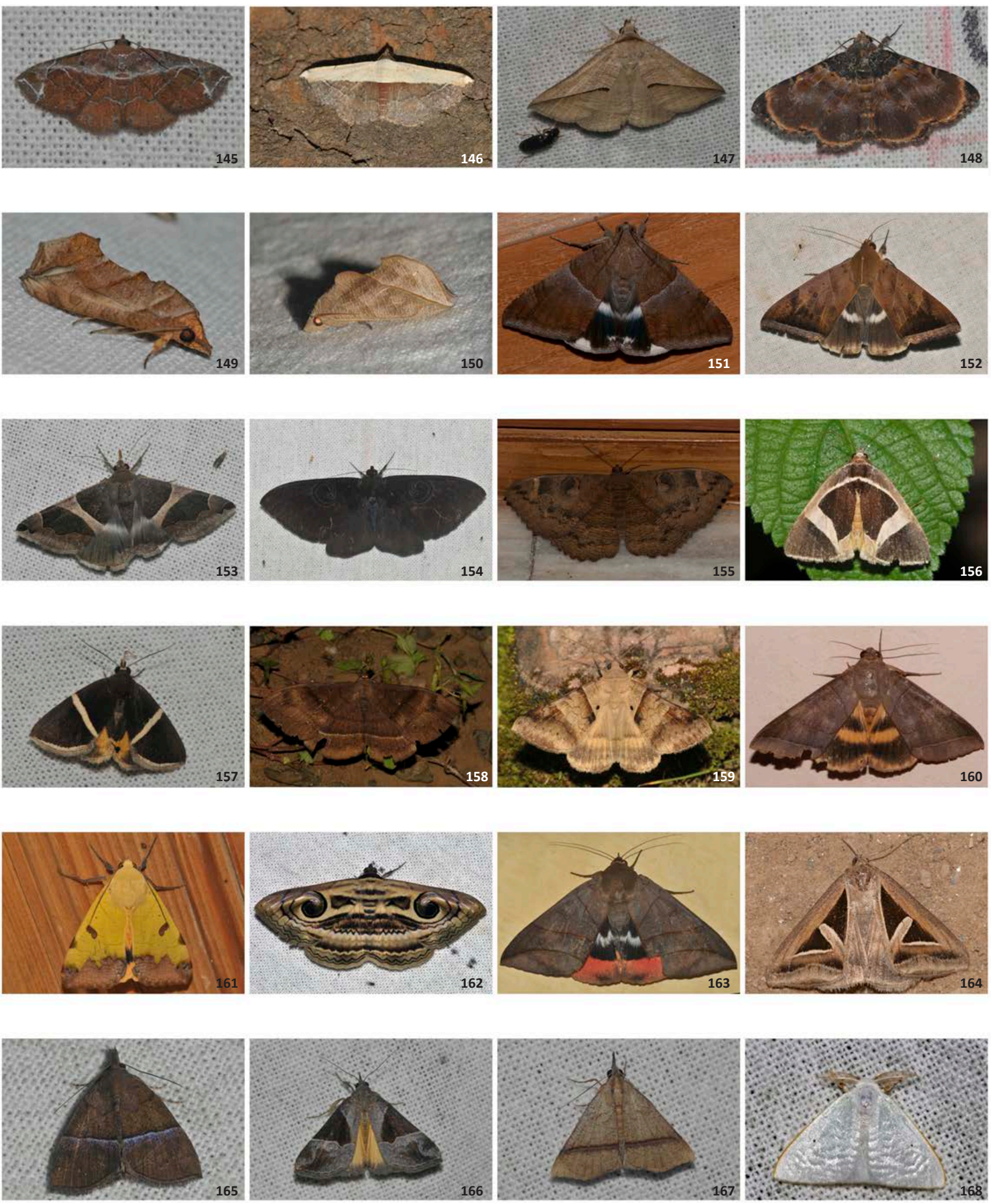

Images 145-168. 145 - Zurobata sp. 2; 146 - Zurobata sp. 3; 147 - Dierna strigata; 148 - Diomea lignocolora; 149 - Oraesia cf. emarginata; 150 - Calyptra cf. parva; 151 - Achaea janata; 152 - Artena dotata; 153 - Dysgonia stuposa; 154 - Erebus caprimulgus; 155 - Erebus hieroglyphica; 156 - Fodina cf. stola; 157 - Fodina pallula; 158 - Lygniodes hypoleuca; 159 - Mocis undata; 160 - Ophiusa coronata; 161 - Ophiusa tirhaca; 162 - Spirama cf. retorta; 163 - Thyas juno; 164 - Trigonodes hyppasia; 165 - Hypena cf. iconicalis; 166 - Hypena quadralis; 167 - Hypena sp.; 168 - Arctornis sp. 1. @) Yash Sondhi 

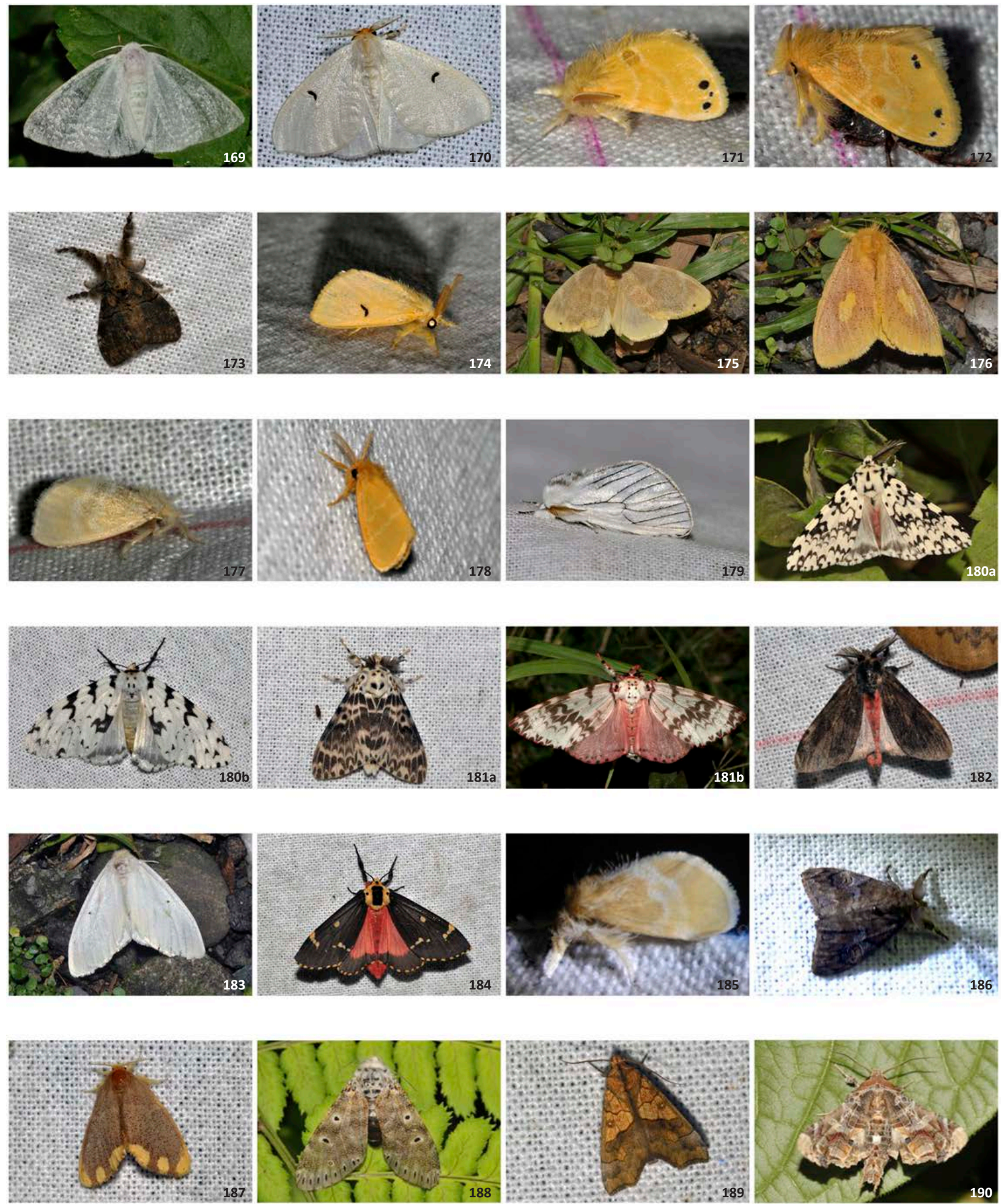

Images 169-190. 169 - Arctornis sp. 2; 170 - Arctornis-I-nigrum; 171 - Artaxa diagramma; 172 - Artaxa guttata; 173 - Dasychira sp.; 174 - Euproctis cf. lunata; 175 - Euproctis cf. vitellina; 176 - Euproctis plagiata; 177 - Euproctis sp. 1; 178 - Euproctis sp. 2;

179 - Himala argentea; 180a - Lymantria cf. concolor (male); 180b - Lymantria cf. concolor (female); 181a - Lymantria mathura (male); 181b - Lymantria mathura (female); 182 -Lymantria obsoleta; 183 - Lymantria sp.; 184 - Lymantria semicincta; 185 -Lymantriades cf. varians; 186 - Orgyia cf. postica; 187 - Somena cf. scintillans; 188 - Varmina indica; 189 - Anomis flava; 190 - Eutelia adulatricoides. (0) Yash Sondhi 

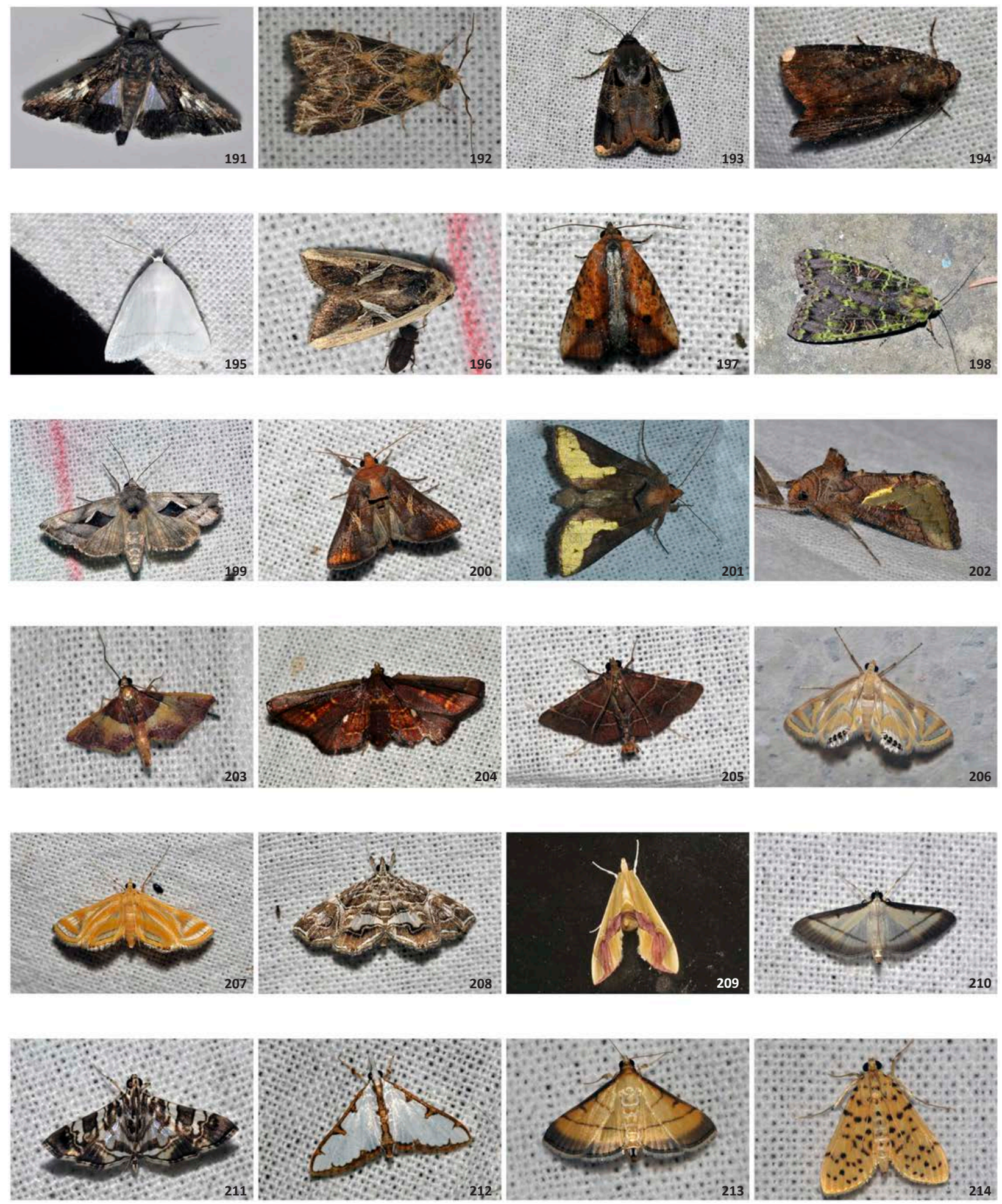

Images 191-214. 191 - Aedia sp.; 192 - Callopistria cf. indica; 193 - Callyna jugaria; 194 - Callyna monoleuca; 195 - Chasmina judicata; 196 - Elaphria cf. conjugata; 197 - Bagada pyrochroma; 198 - Anaplectoides cf. tamsi*; 199 - Macdunnoughia tetragona; 200 - Erythroplusia sp.; 201 - Thysanoplusia intermixta; 202 - Thysanoplusia orichalcea; 203 - Endotricha olivacealis; 204 - Tamaraca torridalis; 205 - Xitha tactilis; 206 - Eoophyla cf. peribocalis; 207 - Eoophyla sp.; 208 - Paracymoriza vagalis; 209 - Agathodes ostentalis; 210 - Bradina diagonalis; 211 - Chabula acamasalis; 212 - Cirrhochrista brizoalis; 213 - Cnaphalocrocis medinalis; 214 - Conogethes punctiferalis. (C) Yash Sondhi 

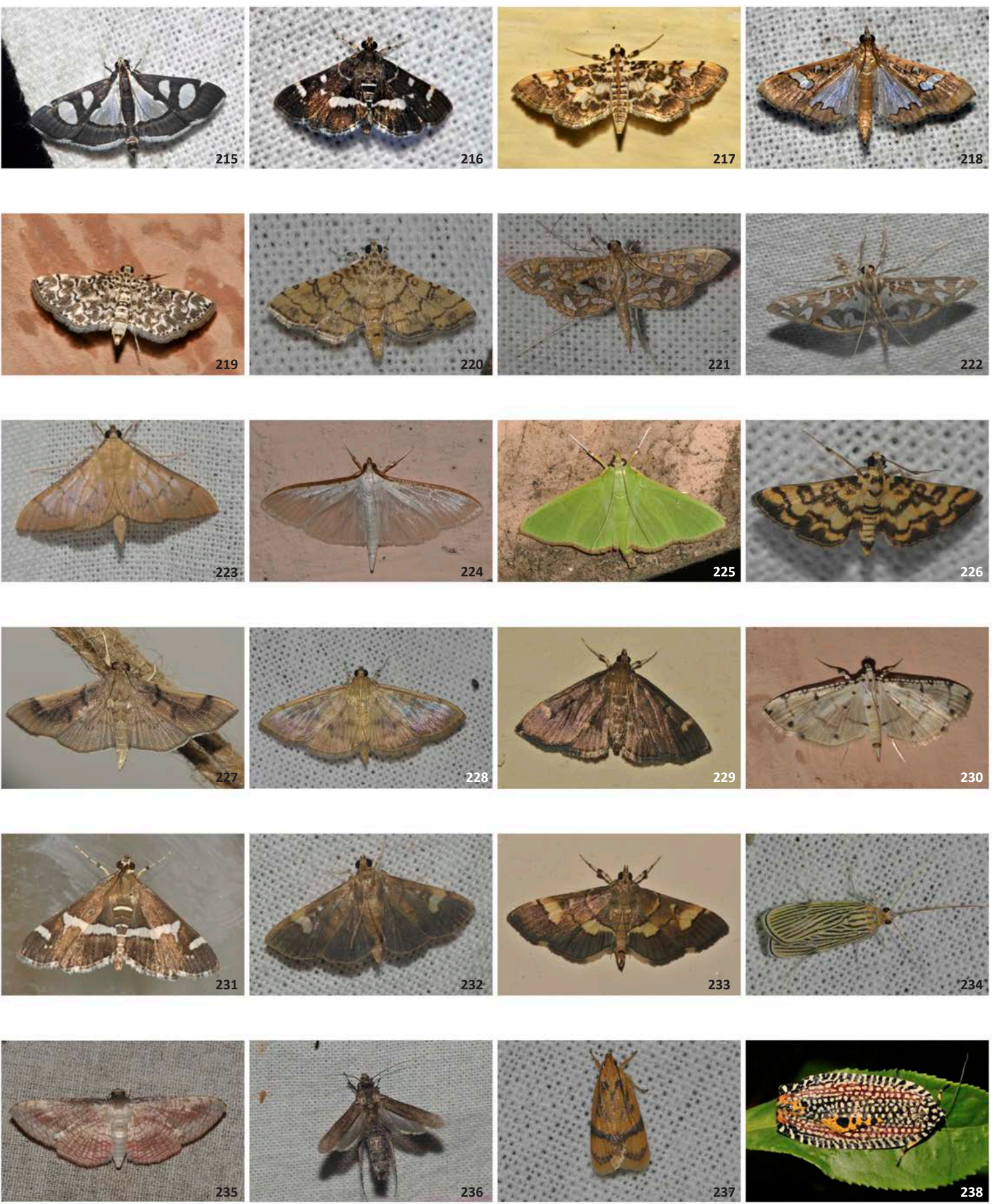

Images 215-238. 215 - Glyphodes bicolor; 216 - Hymenia cf. perspectalis; 217 - Lamprosema commixta; 218 - Maruca cf. vitrata/testulalis; 219 - Metoeca foedalis; 220 - Nacloeia sp.; 221 - Nausinoe cf. geometralis; 222 - Nausinoe perspectata; 223 - Omiodes sp.; 224 - Palpita cf. asiaticalis; 225 - Parotis sp.; 226 - Piletocera cf. chyrsorycta; 227 - Pilocrocis cf. barcalis; 228 - Pleuroptya cf. balteata; 229 - Pleuroptya cf. deficiens; 230 - Pycnarmon cf. cribrata; 231 - Spoladea recurvalis; 232- Syllepte concatenalis; 233 - Syngamia cf. falsidicalis;

234 - Tyspanodes linealis; 235 - Rhodonuera cf. erubrescens; 236 - Gerontha sp.; 237 - Periacma sp.; 238 - Cerace cf. cyanopyga. (c) 238 - Sanjay Sondhi; rest of the images (c) Yash Sondhi 

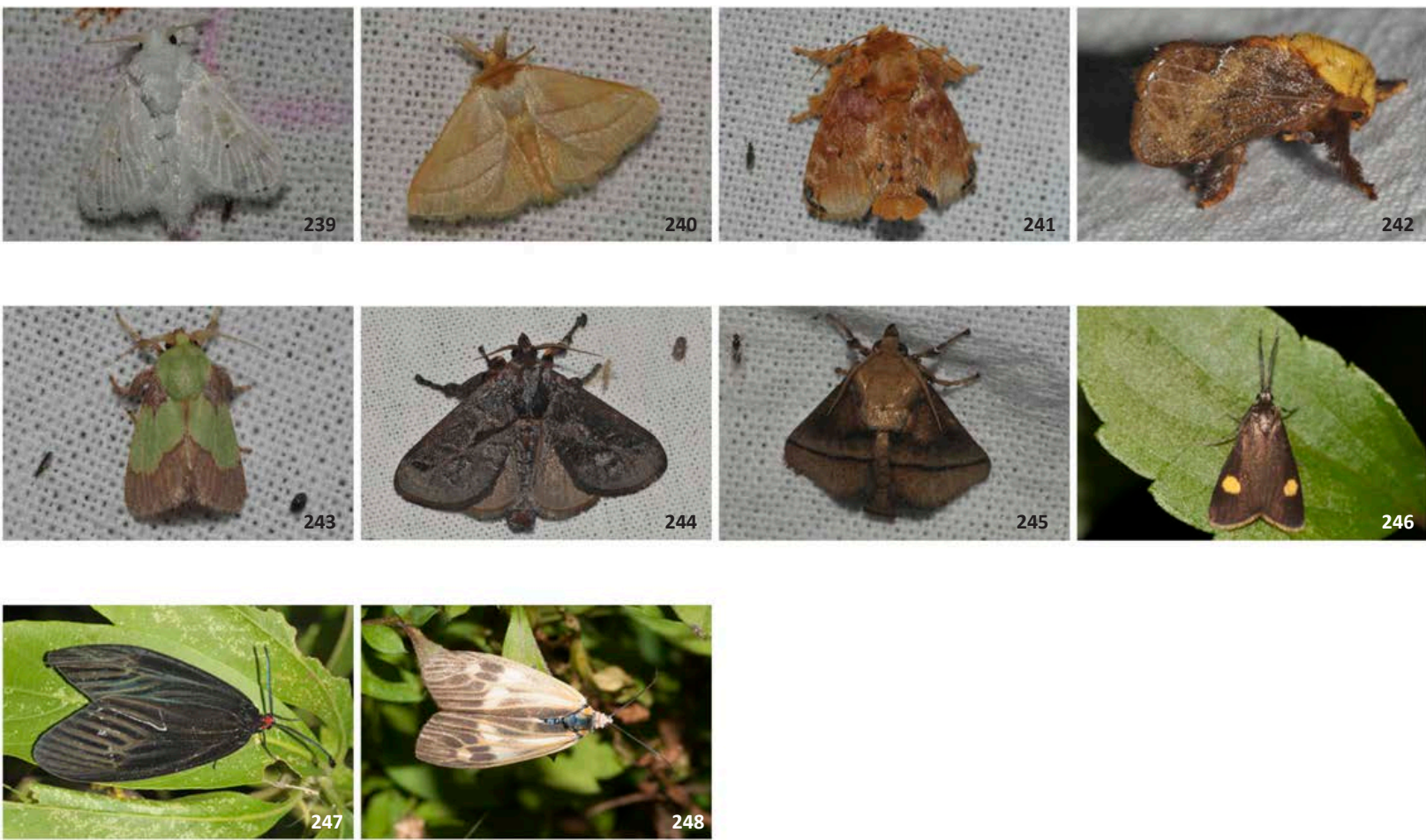

Images 239-248. 239 - Altha subnotata; 240 - Cania cf. bilinea; 241 - Cheromettia cf. apicata; 242 - Miresa sp.; 243 - Parasa hilaris; 244 - Phocoderma velutina; 245 - Thosea sp.; 246 - Brachartona quadrimaculata; 247 - Gynautocera papilionaria; 248 - Soritia pulchella. (ㄷ) 246, 247, 248 - Sanjay Sondhi; rest of the images (c) Yash Sondhi

of India, Dehradun, 237pp.

van Nieukerken E.J., L. Kaila, I.J. Kitching, N.P. Kristensen, D.C. Lees, J. Minet, C. Mitter, M. Mutanen, J.C. Regier, T.J. Simonsen, N. Wahlberg, S.-H. Yen, R. Zahiri, D. Adamski, J. Baixeras, D. Bartsch, B.A. Bengtsson, J.W. Brown, S.R. Bucheli, D.R. Davis, J.D. Prins, W.D. Prins, M.E. Epstein, P. Gentili-Poole, C. Gielis, P. Hättenschwiler, A Hausmann, J.D. Holloway, A. Kallies, O. Karsholt, A.Y. Kawahara, S. Koster, M.V. Kozlov, J.D Lafontaine, G. Lamas, J.F. Landry, S. Lee, M. Nuss, K.-T. Park, C. Penz, J. Rota, A. SchintImeister, B.C. Schmidt,
J.-C. Sohn, M.A. Solis, G.M. Tarmann, A.D. Warren, S. Weller, R.V. Yakovlev, V.V Zolotuhin \& A. Zwick (2011). Order Lepidoptera, pp. 212-221. In: Zhang, Z-Q (ed.). Animal biodiversity: An outline of higher-level classification and survey of taxonomic richness. Zootaxa 3148.

Zolotuhin, V.V. \& A. Pinratana (2005). Moths of Thailand, Volume 4: Lasiocampidae. Brothers of St. Gabriel in Thailand, 209pp+ 46figs+28pls. 